\title{
Atomic clusters with addressable complexity
}

David J. Wales

Citation: The Journal of Chemical Physics 146, 054306 (2017); doi: 10.1063/1.4974838

View online: http://dx.doi.org/10.1063/1.4974838

View Table of Contents: http://aip.scitation.org/toc/jcp/146/5

Published by the American Institute of Physics

\section{Articles you may be interested in}

Inchworm Monte Carlo for exact non-adiabatic dynamics. I. Theory and algorithms

The Journal of Chemical Physics 146, 054105054105 (2017); 10.1063/1.4974328

Announcement: Top reviewers for The Journal of Chemical Physics 2016

The Journal of Chemical Physics 146, 100201100201 (2017); 10.1063/1.4978399

High order path integrals made easy

The Journal of Chemical Physics 145, 234103234103 (2016); 10.1063/1.4971438

Identification of simple reaction coordinates from complex dynamics

The Journal of Chemical Physics 146, 044109044109 (2017); 10.1063/1.4974306

Merging symmetry projection methods with coupled cluster theory: Lessons from the Lipkin model Hamiltonian

The Journal of Chemical Physics 146, 054110054110 (2017); 10.1063/1.4974989

Surface fine structure influence on saturated random packings

The Journal of Chemical Physics 146, 054706054706 (2017); 10.1063/1.4975100

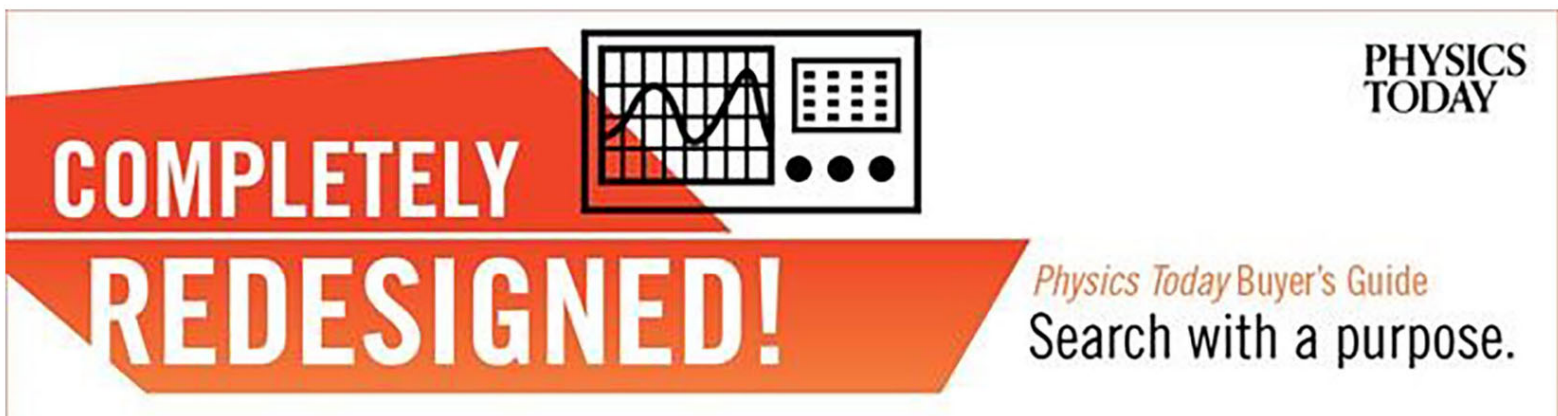




\title{
Atomic clusters with addressable complexity
}

\author{
David J. Wales ${ }^{\text {a) }}$ \\ University Chemical Laboratories, Lensfield Road, Cambridge CB2 1EW, United Kingdom
}

(Received 1 August 2016; accepted 11 January 2017; published online 7 February 2017)

\begin{abstract}
A general formulation for constructing addressable atomic clusters is introduced, based on one or more reference structures. By modifying the well depths in a given interatomic potential in favour of nearest-neighbour interactions that are defined in the reference(s), the potential energy landscape can be biased to make a particular permutational isomer the global minimum. The magnitude of the bias changes the resulting potential energy landscape systematically, providing a framework to produce clusters that should self-organise efficiently into the target structure. These features are illustrated for small systems, where all the relevant local minima and transition states can be identified, and for the low-energy regions of the landscape for larger clusters. For a 55-particle cluster, it is possible to design a target structure from a transition state of the original potential and to retain this structure in a doubly addressable landscape. Disconnectivity graphs based on local minima that have no direct connections to a lower minimum provide a helpful way to visualise the larger databases. These minima correspond to the termini of monotonic sequences, which always proceed downhill in terms of potential energy, and we identify them as a class of biminimum. Multiple copies of the target cluster are treated by adding a repulsive term between particles with the same address to maintain distinguishable targets upon aggregation. By tuning the magnitude of this term, it is possible to create assemblies of the target cluster corresponding to a variety of structures, including rings and chains. Published by AIP Publishing. [http://dx.doi.org/10.1063/1.4974838]
\end{abstract}

\section{INTRODUCTION}

To construct an operational machine, we generally need to assemble a variety of components into a well-defined spatial arrangement. The experimental realisation ${ }^{1}$ of programmed self-assembly for a structure composed of thousands of distinct building blocks has therefore generated great interest. Here the building blocks are "DNA bricks," which can bind by hybridisation to four neighbours. The resulting assemblies are considered "addressable," in that the different components are located in specific local environments. Understanding and developing design principles for such structures could provide a route to translation of information encoded in nanoscale building blocks into new materials with a specific structure and function. Developing models that reproduce the key experimental results using the simplest possible representations is therefore an important challenge, and initial efforts for DNA bricks have already reproduced addressable assembly for as many as 1000 distinct components. ${ }^{2}$ Recent insight into computer simulation indicates that robust self-assembly may require precise conditions for nucleation to occur. The yield for the target structure can be improved significantly using a specific annealing protocol. ${ }^{3}$

Clusters of colloidal particles, functioning as pseudoatoms, also hold the prospect of designed addressable structures. ${ }^{4,5}$ Direct observation of structure, dynamics, and thermodynamics can be obtained or inferred from optical microscopy for building blocks composed of polystyrene

a)dw34@cam.ac.uk microspheres, inspiring complementary theoretical analysis. ${ }^{6-9}$ Design principles have been considered for these clusters, ${ }^{10}$ including an approach ${ }^{7}$ based on the random energy model $^{11}$ for protein folding. ${ }^{12}$ The latter results suggest that optimal yields for self-assembly may be obtained when the nearest-neighbour interactions between different components are of comparable strength. ${ }^{7}$

In the present contribution, a general framework for constructing an addressable interatomic potential is suggested, which uses the pairwise Lennard-Jones form ${ }^{13}$ for specificity,

$$
V=4 \sum_{i<j} \epsilon_{i j}\left[\left(\frac{\sigma}{r_{i j}}\right)^{12}-\left(\frac{\sigma}{r_{i j}}\right)^{6}\right],
$$

where $r_{i j}$ is the distance between atoms $i$ and $j$. Here the well depth is employed to produce addressable functionality. As for structure-based potentials and Gō models, ${ }^{14}$ we introduce a bias towards a particular local minimum, in this case a specific permutational isomer of a selected stationary point. In the present work, we simply define

$$
\epsilon_{i j}= \begin{cases}\epsilon, & r_{i j}^{0} \leq r_{c}, \\ \alpha \epsilon, & r_{i j}^{0}>r_{c},\end{cases}
$$

where $0 \leq \alpha \leq 1, r_{i j}^{0}$ is the distance between atoms $i$ and $j$ in the reference structure, indicated by the superscript 0 , and $r_{c}$ is a cutoff. Analysing the effect of $\alpha$ on the underlying landscape is the main objective of the present work. $\epsilon$ and $\sigma$ are chosen as the units of energy and distance, and $r_{c}$ was set to 1.2 for all the calculations reported below. Hence pair interactions that do not correspond to nearest neighbours in the reference structure are disfavoured as $\alpha$ is reduced from unity. 
Biasing the potential with reference to a particular configuration has been considered before in studies of glassy systems, including structural glass formers ${ }^{15,16}$ and spin models. ${ }^{17}$ The potential is shifted according to the overlap with a given reference configuration, defined in terms of a sum of step functions over all distinct pairs of atoms for the atomic models. The principal difference from the present work is that the addressable potential defined above is based on a particular permutational isomer. This choice partly reflects alternative theoretical perspectives, where models based upon density are often used in treating condensed matter and glasses, while a single-particle view is usually adopted for clusters and finite systems. Overlap functions have also proved useful in the studies of glass forming systems that employ pinned (frozen) particles ${ }^{18-27}$ and cavities defined by a frozen atomic environment. ${ }^{28-37}$

There are some similarities between the addressable potential defined in Equation (2) and elastic network models ${ }^{38}$ designed to treat conformational changes in biomolecules. ${ }^{39-56}$ The common theme is identification of nearest neighbours, but the network models usually implement this structure through local harmonic springs. Doublewell Gō models have also been defined for biomolecules, ${ }^{57-61}$ where switching is achieved between parameterisations biased towards distinct minima.

In this report, we construct a doubly addressable potential by assigning

$$
\epsilon_{i j}= \begin{cases}\epsilon, & \min \left(r_{i j}^{0}, r_{i j}^{1}\right) \leq r_{c}, \\ \alpha \epsilon, & \min \left(r_{i j}^{0}, r_{i j}^{1}\right)>r_{c},\end{cases}
$$

using the minimum pair distance for two reference structures, 0 and 1. This formulation is similar in spirit to that of Murugan et al., ${ }^{62}$ who considered "multifarious" structures composed of a variety of components. In the latter model, favourable pair interactions were included for components that needed to interact in any of a number of target structures. The capability to select alternative targets was then demonstrated using different (super)critical nucleation seeds. The potential considered in the present work, defined in Equation (3), defines two targets in a similar way. The focus here is on the appearance of the underlying energy landscape, especially in terms of the self-assembly characteristics defined by funnelling properties. For the doubly addressable potential, it might be possible to select between the two alternatives using a kinetic approach based on nucleation, especially for the example considered where the underlying packing is very different. Alternatively, with some further modification, alternative structures might be selected using external parameters, such as an applied field. ${ }^{63}$ Experimentally, addressable self-assembly usually involves formation of multiple copies of the target structure, for example, from a solution of DNA strands. DNA-based materials have been used extensively in previous experiments, for functionalised polymer microspheres ${ }^{64-66}$ and gold particles, ${ }^{67-70}$ DNA scaffold and staple systems, ${ }^{71,72}$ and single-stranded tiles and bricks. ${ }^{1,73,74}$ Alternatively, some recent advances have focused upon self-assembly of synthetic peptides. ${ }^{75,76}$ The present model is intended to be as general as possible and might help guide experiments based on a variety of building blocks.
Establishing how the energy landscape depends upon parameters of the model potential for a single incarnation of the target structure provides the foundations for treating multiple copies. For a target containing $N$ particles, we can extend the formulation of the potential using the function $D(i, N)=\operatorname{Mod}(i-1, N)+1$, where $\operatorname{Mod}(i-1, N)$ is the particle index modulo $N$, ranging from 0 to $N-1$. The function $D(i, N)$ therefore produces an address label in the range 1 to $N$, and we choose

$$
\epsilon_{i j}= \begin{cases}\epsilon, & r_{D(i, N) D(j, N)}^{0} \leq r_{c}, \\ \alpha \epsilon, & r_{D(i, N) D(j, N)}^{0}>r_{c}, \\ 0, & D(i, N)=D(j, N),\end{cases}
$$

with an additional repulsive term between particles that share the same address,

$$
V^{\mathrm{rep}}=4 \sum_{i<j} \epsilon^{\mathrm{rep}}\left(\frac{\sigma}{r_{i j}}\right)^{12} \delta[D(i, N), D(j, N)],
$$

where $\delta[k, l]$ is the Kronecker delta, equal to one if $k=l$ and zero otherwise. This formulation is invariant to the exchange of particles with the same address.

Preliminary results for systems containing multiple copies of the target cluster are presented in Section V. If $\epsilon^{\text {rep }}$ is too small, the target clusters can aggregate to produce conglomerates where the individual clusters are distorted or difficult to distinguish. However, as $\epsilon^{\text {rep }}$ increases, the favoured structures for the aggregates contain well-defined copies of the target cluster, assuming that we have an equal number of particles for each of the $N$ address labels. The global minimum changes as a function of $\epsilon^{\text {rep }}$, passing through stacked and single rings of clusters (Section V).

The formulation in Equation (5) was chosen because it is a simple and convenient way to tune the structures supported by the model. The aim is to understand the minimal conditions on the potential required to realise these structures. To make contact with particular experiments, it will be necessary to map the interparticle forces that are under experimental control into the model, and vice versa. The increasing capabilities to tune interactions between building blocks based upon DNA and colloids suggest that it will be possible to exploit this route to treat specific applications.

The principal advantage of the present model is that it enables us to analyse the organisation of the underlying energy landscape in detail and predict changes in the likely self-assembly characteristics in terms of the strength of the competing interactions. For example, we can tune the energy scales associated with higher energy structures containing wrongly addressed particles within each $N$-body cluster, and the barriers between alternative packings of the target clusters within aggregates. The resulting energy landscapes can be analysed in terms of local rearrangements within each cluster, and exchanges of particle with the same address between different clusters. Initial results are reported in Section V.

\section{METHODS}

All the methodology employed in the present work for exploring the potential energy landscape and constructing 
kinetic transition networks ${ }^{77-79}$ is well established and has been described in detail before. In brief, the characterisation of each system begins with a basin-hopping global optimisation $^{80-82}$ survey, which harvests low-lying minima along with the global minimum. For the small clusters considered here, we can find all the distinct minima in this stage, and we then attempt to connect them pairwise using the doubly-nudged ${ }^{83}$ elastic band ${ }^{84,85}$ (DNEB) approach, which gives most, if not all, of the distinct transition states after the refinement of candidate structures using hybrid eigenvector-following. ${ }^{86-88}$ Here we define transition states geometrically, as saddle points of index one, with a single negative Hessian eigenvalue. ${ }^{89}$ The connection attempts were repeated for different DNEB parameters, such as image and iteration density, until no new transition states were obtained. Cross-relaxation checks were conducted for the databases constructed with different values of the well-depth parameter $\alpha$, as described in Section III. The databases are visualised using standard constructions for disconnectivity graphs. ${ }^{90,91}$

For the larger clusters, containing 13 and 55 particles, we can only expect to converge the landscape in the region of the global minimum. Here we refined stationary point databases using various tools developed within the discrete path sampling ${ }^{92,93}$ framework, as implemented in the PATHSAMPLE program. ${ }^{94}$

\section{RESULTS}

The energy landscapes were characterised for individual target clusters of 5, 6, 7, 13, and 55 atoms. For $5 \leq N \leq 7$, complete enumeration of all the minima and transition states should be feasible. Starting from the stationary points for $\alpha=1$, all the permutation-inversion isomers were constructed and then relaxed for successive values of $\alpha=0.75,0.5$, and 0.25 using LBFGS minimisation for the eigenvectorfollowing for the transition states, ${ }^{86}$ as described above. LBFGS is a limited memory version of the quasi-Newton Broyden, ${ }^{95}$ Fletcher, ${ }^{96}$ Goldfarb,${ }^{97}$ Shanno, ${ }^{98}$ BFGS algorithm. Permutation-inversion isomers are structures that cannot be superimposed by an overall rotation, but differ only through the arrangement of atoms of the same element, which may be combined with inversion of all particle coordinates through the origin.

As a cross-check, the databases obtained for each $\alpha$ were subsequently relaxed for all the other $\alpha$ values considered, and this process was repeated until no new stationary points were obtained. Relatively small maximum step sizes were employed in all the geometry optimisations to relax structures slowly. A convergence condition of $10^{-10}$ reduced units for the root mean square gradient was adopted, for which energy differences of $10^{-13}$ or more could be distinguished.

In each case, results were obtained for a particular permutation-inversion isomer of the global minimum as the reference. These systems will be denoted by a $*$ superscript, e.g., $\mathrm{LJ}_{13}^{*}$ for an icosahedral reference geometry with 13 atoms. For $\mathrm{LJ}_{55}$ two additional potentials were considered, one for a transition state of $O_{h}$ symmetry as the reference, denoted $\mathrm{LJ}_{55}^{O_{h}}$, and a doubly addressable system with the two reference geometries corresponding to isomers of the $I_{h}$ global minimum and the $O_{h}$ transition state. The cuboctahedral transition state links two permutational isomers of the Mackay ${ }^{99}$ icosahedron via a concerted sextuple diamondsquare-diamond ${ }^{100}$ rearrangement. ${ }^{101}$ Hence these experiments test our ability to stabilise a structure that corresponds to a saddle point for the original potential and probe the conditions required to construct a doubly addressable system.

\section{A. Results for $L J_{5}^{*}$}

If permutation-inversion isomers are not distinguished, then the $\mathrm{LJ}_{5}$ cluster has a single minimum and two distinct transition states. The transition states link permutational isomers of the minimum via degenerate rearrangements. ${ }^{102}$ The number of permutation-inversion minima corresponding to distinct wells on the potential energy surface is $2 N ! / o_{\alpha}$ for $N$ identical atoms and a structure with point group order $o_{\alpha} \cdot{ }^{9,102-106}$ Hence there are $2 \times 5 ! / 12=20$ versions of the $D_{3 h}$ minimum and 30 and 60 distinct versions of the two transition states (Table I).

The simple choice of addressable potential employed in the present work, with just two distinct well depths, does not resolve all the degeneracies in energy. Instead, the 20 minima split into sets of 2,6 , and 12 , as shown in the disconnectivity graphs in Figure 1. The splitting between the two larger sets varies from $2.8 \times 10^{-5}$ for $\alpha=0.75$ to $2.3 \times 10^{-4}$ for $\alpha=0.25$, values that are too small to discern in the graphs. Similarly, the transition states split into three sets of 6 , four sets of 12 , and one set of 24, preserving the total of 90 . The remaining permutational degeneracy could be lifted by adding a random component to the pair well depths, but this step does not seem helpful in the present study.

The results in Figure 1 illustrate two general points. First, the potential defined in Equation (1) has inversion symmetry, and this two-fold degeneracy is evident in the disconnectivity graphs in the left panels of the figure. Second, the landscape becomes increasingly biased towards the reference structure as $\alpha$ decreases, realising the design principles on which this general addressable form is based. The trend is clearer when inversion isomers are lumped together, as shown in the right hand panels of Figure 1, and this representation is retained for all the graphs that follow.

TABLE I. The number of distinct structures and permutation-inversion isomers corresponding to minima and transition states for addressable LennardJones clusters as a function of the bias parameter $\alpha$.

\begin{tabular}{|c|c|c|c|c|c|}
\hline \multirow[b]{2}{*}{ Cluster } & \multirow[b]{2}{*}{$\alpha$} & \multicolumn{2}{|c|}{ Structures } & \multicolumn{2}{|c|}{ Permutation-inversion isomers } \\
\hline & & Minima & Transition states & Minima & Transition states \\
\hline $\mathrm{LJ}_{5}$ & 1.00 & 1 & 2 & 20 & 90 \\
\hline $\mathrm{LJ}_{5}$ & 0.75 & 3 & 8 & 20 & 90 \\
\hline $\mathrm{LJ}_{5}$ & 0.50 & 3 & 8 & 20 & 90 \\
\hline $\mathrm{LJ}_{5}$ & 0.25 & 3 & 8 & 20 & 90 \\
\hline $\mathrm{LJ}_{6}$ & 1.00 & 2 & 3 & 390 & 1800 \\
\hline $\mathrm{LJ}_{6}$ & 0.75 & 10 & 26 & 390 & 1896 \\
\hline $\mathrm{LJ}_{6}$ & 0.50 & 10 & 27 & 390 & 1944 \\
\hline $\mathrm{LJ}_{6}$ & 0.25 & 9 & 24 & 366 & 1776 \\
\hline $\mathrm{LJ}_{7}$ & 1.00 & 4 & 12 & 8904 & 63840 \\
\hline $\mathrm{LJ}_{7}$ & 0.75 & 248 & 1226 & 8904 & 62320 \\
\hline $\mathrm{LJ}_{7}$ & 0.50 & 248 & 1549 & 8904 & 59418 \\
\hline $\mathrm{LJ}_{7}$ & 0.25 & 230 & 1226 & 8184 & 47022 \\
\hline
\end{tabular}




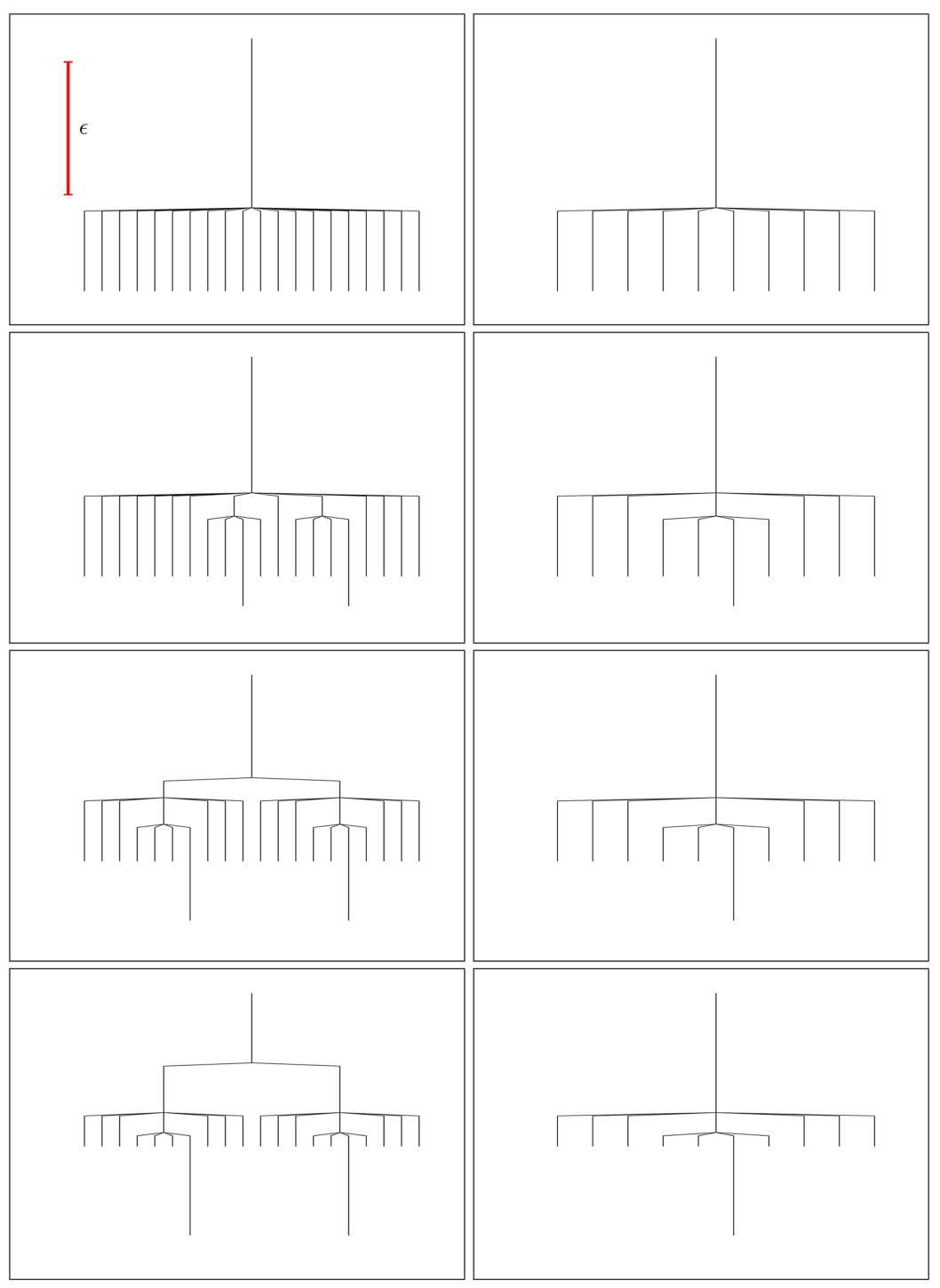

FIG. 1. Disconnectivity graphs for addressable $\mathrm{LJ}_{5}$ with $\alpha$ values of $1,0.75,0.5$, and 0.25 (top to bottom). In the left column, all distinct permutation-inversion isomers are included, while in the right column, pairs of inversion isomers are lumped together. The scale and energy range are the same for all panels.

\section{B. Results for $L_{6}^{*}$}

For $\mathrm{LJ}_{6}$ there are 30 distinct permutation-inversion isomers of the octahedral global minimum and 360 versions of the $C_{2 v}$ local minimum. There are three different transition state structures, with 720 versions for two of them and 360 versions for the third. For $\alpha=0.75$ and 0.5 , the octahedral minima split into two sets with point group order 48, twelve of order 8 , and sixteen of order 6 , to preserve the total of 30 . The two minima with $O_{h}$ symmetry correspond to the permutational isomer chosen as the reference and its mirror image. The sets of twelve and sixteen minima have atoms exchanged in one of the twelve edges of the octahedron or three atoms permuted in a face. There are two possible permutations for each of eight faces, producing sixteen distinguishable minima. The splittings that occur for lower symmetry minima and transition states can also be analysed in this way, but details are omitted for brevity.

Table I summarises the results for the smaller clusters as a function of $\alpha$. Here it is noteworthy that the number of stationary points can change when $\alpha \neq 1$, and an isomorphism with the unperturbed system need not be preserved. The disconnectivity graphs for $\mathrm{LJ}_{6}^{*}$ in Figure 2 lump inversion isomers together and illustrate how the complexity of the landscape increases combinatorially with system size. The addressable potential produces a well-defined global minimum as $\alpha$ decreases.

\section{Results for $L_{7}$}

For $\mathrm{LJ}_{7}$ the $D_{5 h}$ global minimum (pentagonal bipyramid) has 504 distinct permutation-inversion isomers. Two minima with point group order 6 support 1680 versions each, with 5040 for the remaining $C_{2}$ minimum, giving a total of 8904 . For the twelve distinct transition states identified, there is one with point group $D_{3 d}$, three with point group $C_{2 v}$, five with point group $C_{s}$, and three with point group $C_{1}$. The total number of permutation-inversion isomers is therefore $840+3 \times 2520$ $+5 \times 5040+3 \times 10080=63840$.

Even when inversion isomers are lumped together, the details of the corresponding disconnectivity graphs are difficult to distinguish (Figure 3), although the emergence of an addressable global minimum for smaller $\alpha$ is still clear. The resulting landscape has the form that we associate with the efficient self-organisation. The "palm tree" structure ${ }^{91,102,107}$ corresponds to a well-defined global potential energy minimum 


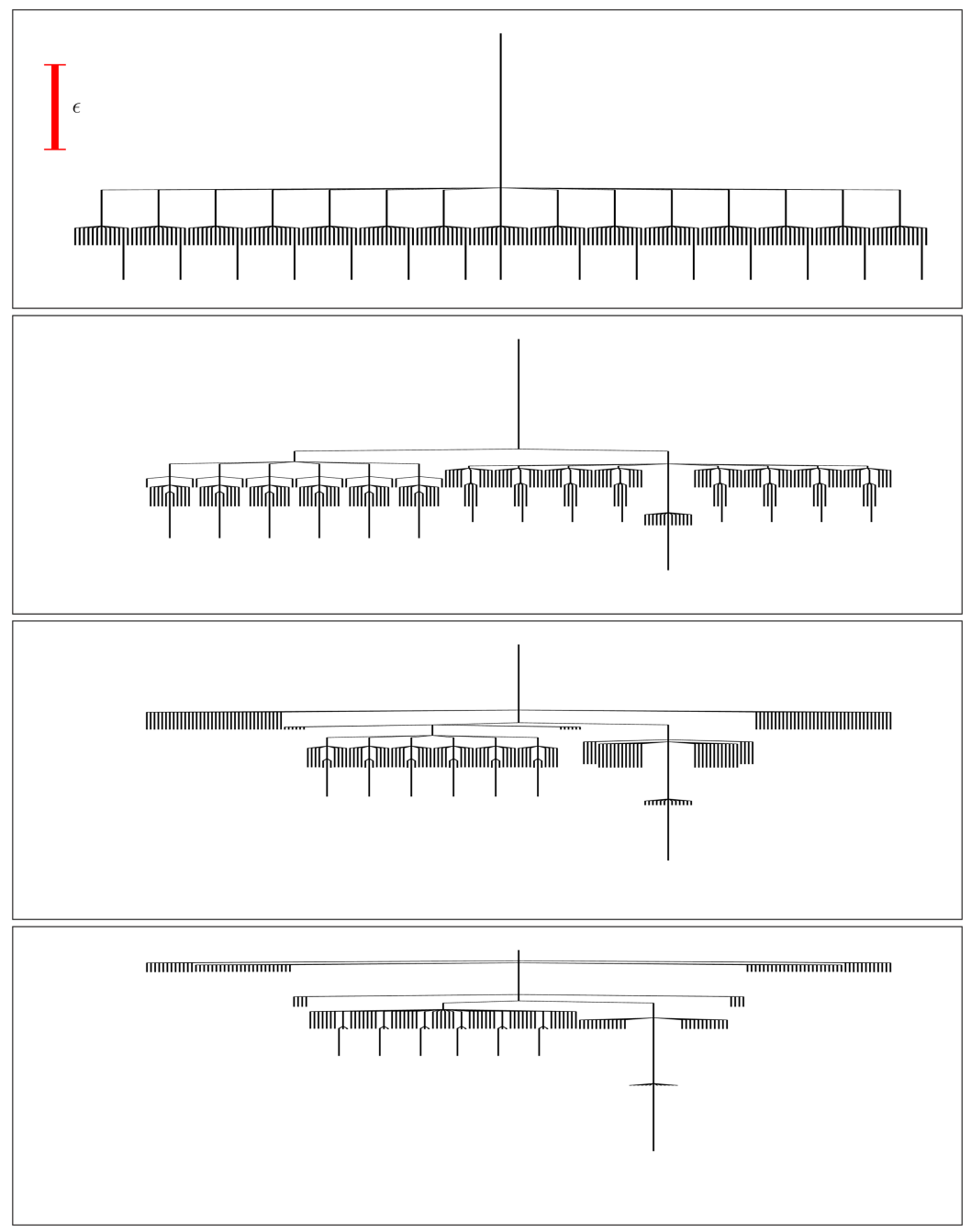

FIG. 2. Disconnectivity graphs for addressable $\mathrm{LJ}_{6}$ for $\alpha$ values of $1,0.75,0.5$, and 0.25 (top to bottom). All distinct permutational isomers are included; inversion isomers are lumped together. The scale and energy range are the same for all panels. with no other low-lying structures separated by high barriers, which would constitute kinetic traps. The resulting free energy minimum should therefore be kinetically accessible over a wide range of temperature, ${ }^{12,108}$ producing a landscape with "funnelling" properties ${ }^{109-111}$ in terms of convergent kinetic pathways. ${ }^{112}$ The landscape entropy, ${ }^{113-115}$ defined in terms of the energy density of local minima, decreases systematically on downhill paths to the global minimum.

Further coarse-graining of the landscape may help visualise the above properties more clearly. Minima with no direct connections to a lower energy minimum lie at the terminus of a monotonic sequence ${ }^{116-119}$ where it is not possible to reach a lower minimum via a rearrangement corresponding to a single transition state. The disconnectivity graph including only these minima provides a simplified view of the landscape that preserves the structure and connectivity of the monotonic sequence basins. An example is shown in Figure 4 for $\mathrm{LJ}_{7}^{*}$ with $\alpha=0.25$. The 14 minima in the resulting graph are all permutational isomers of the global minimum pentagonal bipyramid. The next seven minima above the global minimum correspond to a pair exchange of adjacent equatorial atoms, or exchange of an axial and an equatorial atom, defining a set of five and a set of two degenerate configurations, with very similar energies. In each case, two nearest-neighbour contacts in the reference structure are lost, resulting in an energy increase of approximately $2 \alpha$ (in units of $\epsilon$ ). The next set of five minima lies about $3 \alpha$ above the global minimum, with three nearest-neighbour contacts lost within the equatorial set of atoms. All five contacts in the equator are lost in the highest minimum, and this isomer is unique aside from the corresponding permutation-inversion isomer. In each case, the permutation-inversion isomers have again been lumped together.

The structures corresponding to monotonic sequence termini are actually a form of biminimum, as defined in recent work on global optimisation for multicomponent systems. ${ }^{120,121}$ For nanoalloy clusters, a biminimum is defined as a local minimum whose energy cannot be lowered by interchanging any inequivalent particles and reminimising. We can generalise this idea to multiminima, ${ }^{121,122}$ where the energy cannot be lowered further by perturbations in any number of different metric spaces, including continuous coordinate space, and identity swaps for different atoms or exchanges in some other category. Hence the monotonic sequence termini qualify as biminima because the energy cannot be lowered by stepping to any adjacent minimum that is connected by a single transition state. This viewpoint can be very helpful for interpreting the disconnectivity graphs that retain only the corresponding subset of local minima, which is a convenient way to provide a visualisation of large databases. 


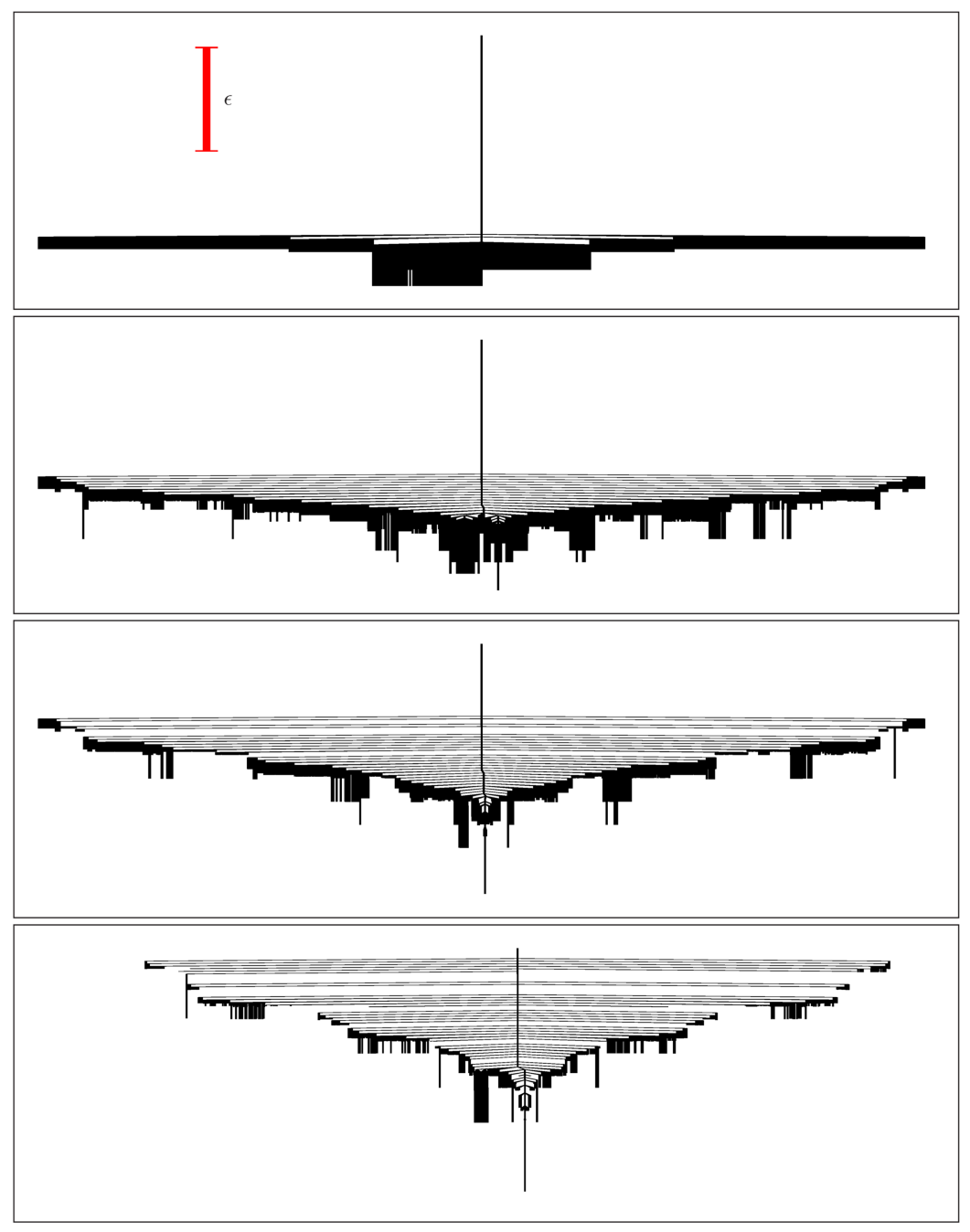

FIG. 3. Disconnectivity graphs for addressable $\mathrm{LJ}_{7}$ for $\alpha$ values of $1,0.75,0.5$, and 0.25 (top to bottom). All distinct permutational isomers are included; inversion isomers are lumped together. The scale and energy range are the same for all panels.
The underlying structure that is revealed can provide important insight into the emergence of observable properties, such as multiple relaxation time scales and features in the heat capacity. ${ }^{92,93,123-128}$

\section{Results for $\mathrm{LJ}_{13}^{*}$}

For $\mathrm{LJ}_{13}^{*}$ we choose to present the landscape for $r_{c}=1.2$ and $\alpha=0.25$, which exhibits a well-defined self-organising structure. In contrast to the smaller clusters, above, we can sample only a small fraction of the stationary points for this system, and the focus is on converging the database in the low energy range of interest. To examine global properties, such as the heat capacity, at higher temperatures would require additional calculations to provide a proper representation of higher energy minima corresponding to the liquid-like phase. We plan to employ basin-sampling ${ }^{115}$ for this purpose in future work.

Two disconnectivity graph representations of the potential energy landscape are compared in Figure 5, and characteristics of the connected component of the database that includes the global minimum are summarised in Table II. The panel on the left includes only minima in the lowest 2000 that correspond to monotonic sequence termini, while the graph on the right includes the lowest 500 minima. Some of the latter structures do not correspond to the biminima defined by monotonic sequence termini because they are directly connected to a lower energy structure, so they do not appear in the left panel. The two graphs are complementary: for example, the secondlowest minima are not present in the left panel because they are connected to the global minimum. However, the structure of this part of the landscape is clear because these minima correspond to the exchange of two atoms in each of the thirty edges of the icosahedron. Both representations correspond to the organisation we would associate with efficient relaxation to the global minimum, again suggesting a successful addressable design.

\section{E. Results for $L J_{55}^{I_{h}}, L J_{55}^{O_{h}}$, and $L J_{55}^{I_{h}+O_{h}}$}

For this larger cluster, two additional tests were conducted to investigate the addressability of a target structure corresponding to a higher energy stationary point (in this case a transition state) and a doubly addressable potential that targets two reference geometries. The capability to select different morphologies, and to design multifunnel landscapes, could play a particularly important role in future design of multifunctional landscapes ${ }^{129}$ and molecular switches, such as rings and cages that might open and close in response to external conditions. ${ }^{63}$

The double reference potential defined in Equation (3) can be made more flexible using different cutoffs and well 

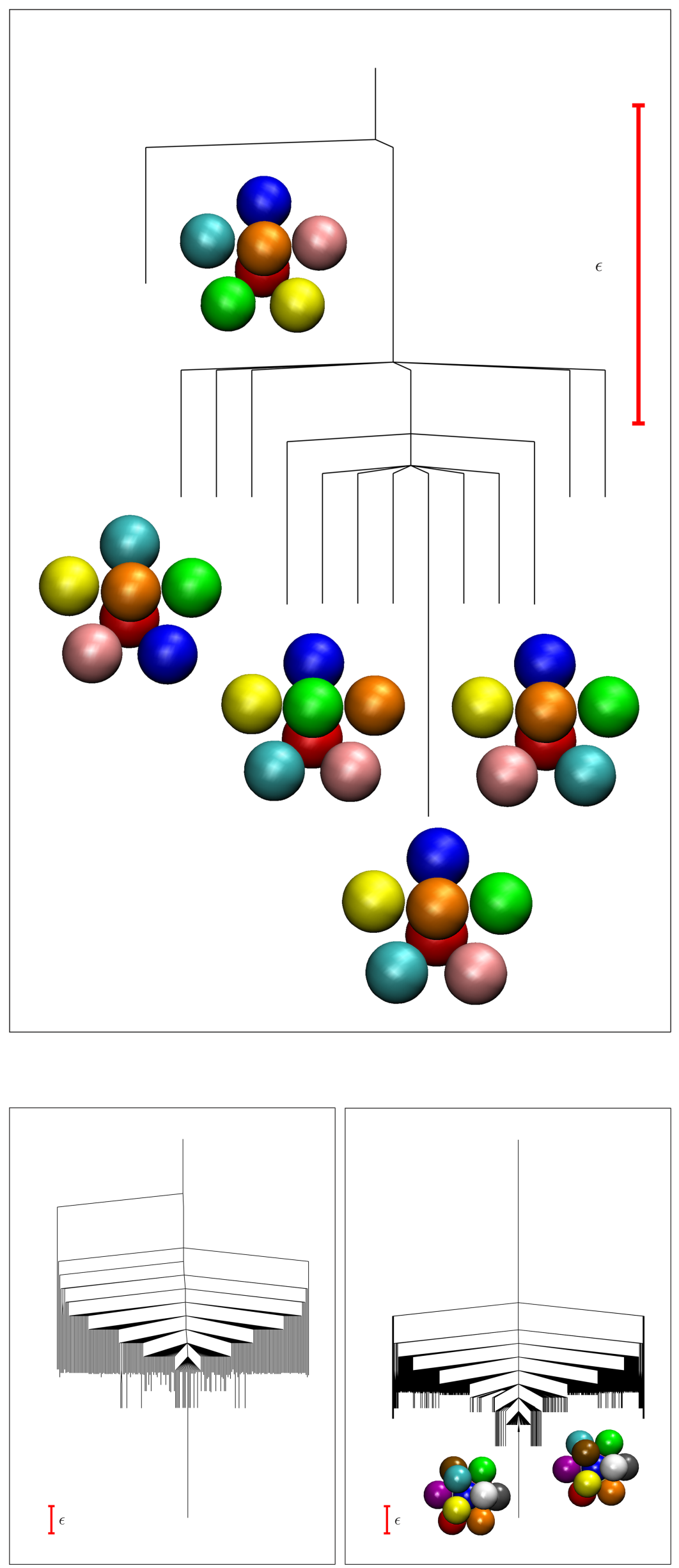

FIG. 4. Disconnectivity graph for addressable $\mathrm{LJ}_{7}$ with $\alpha=0.25$ showing only minima that correspond to monotonic sequence basin bottoms. The relative scale and energy range are the same as for the panels in Figure 3. One representative of each set of minima is shown using a consistent shading for the atoms.

FIG. 5. Disconnectivity graphs for addressable $\mathrm{LJ}_{13}$ with $\alpha=0.25$ using an isomer of the icosahedral global minimum as the reference. Inversion isomers are lumped together. Left: only minima that correspond to monotonic sequence basin bottoms in the lowest 2000 are included. Right: the lowest 500 minima, with the structures of the global minimum and one permutational isomer of the second-lowest minima illustrated. Thirty second-lowest minima have two neighbours swapped in one of the thirty edges in the outer shell. 
TABLE II. The number of permutation isomers in the databases corresponding to minima and transition states for addressable Lennard-Jones clusters containing 13 and 55 atoms. These values include only stationary points belonging to the connected component of the database that includes the global minimum. Sampling was continued for these sizes until the low-energy region of the disconnectivity graphs appeared to have converged.

\begin{tabular}{lccc}
\hline \hline Cluster & $\alpha$ & Minima & Transition states \\
\hline $\mathrm{LJ}_{13}^{*}$ & 0.25 & 396223 & 504668 \\
$\mathrm{LJ}_{55}^{I_{h}}$ & 0.20 & 7893 & 12012 \\
$\mathrm{LJ}_{55}^{O_{h}}$ & 0.20 & 19439 & 29915 \\
$\mathrm{LJ}_{55}^{I_{h}+O_{h}}$ & 0.20 & 47022 & 68886 \\
\hline \hline
\end{tabular}

depths for the two references. To locate suitable parameters, the pathway mediated by the $O_{h}$ transition state between two $I_{h}$ minima was analysed systematically, first to stabilise the transition state as a minimum, and then to adjust the two target structures to have comparable potential energy. Using cutoffs of 1.2 for both references produces a range of $\alpha$ values where both targets correspond to minima, and choosing $\alpha=0.2$ gives a pathway between these minima mediated by a single transition state of lower symmetry, with similar barriers in the two directions. Hence the present investigation did not require additional flexibility beyond the form of Equation (3).

For each system, $\mathrm{LJ}_{55}^{I_{h}}, \mathrm{LJ}_{55}^{O_{h}}$, and $\mathrm{LJ}_{55}^{I_{h}+O_{h}}$, stationary point databases were expanded until the appearance of the disconnectivity graphs in the low energy range of interest ceased to change significantly. The number of minima and transition states in the connected component of each database containing the global minimum is given in Table II, and the disconnectivity graphs retaining monotonic sequence termini that lie in the lowest 2000 minima are shown in Figure 6. In each case, the second-lowest minimum is added to this set for comparison.

These results show that the simple addressable potentials defined in Section I can indeed selectively stabilise different morphologies, which need not be local minima for the original potential. Furthermore, the framework can be extended to multiple targets in a straightforward way. Comparison of the low-lying regions of the potential energy landscapes for the three systems in Figure 6 suggests some further trends, which need to be tested in future work to determine if they are generally applicable. Specifically, the addressable landscape is simplest when the target structure is a permutational isomer of the global potential minimum

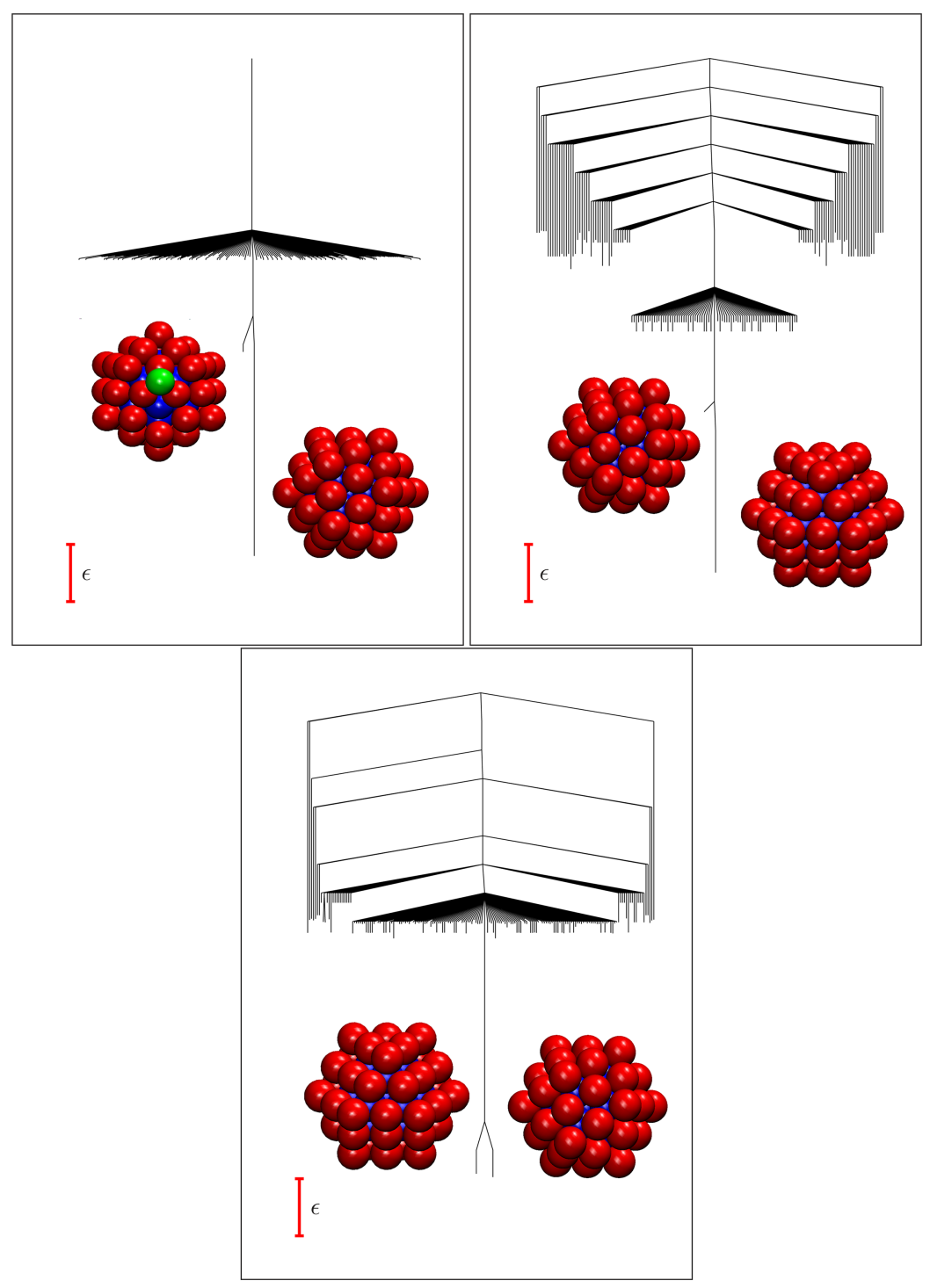

FIG. 6. Disconnectivity graphs for addressable $\mathrm{LJ}_{55}$ clusters with $\alpha=0.2$ using the icosahedral global minimum as the reference (top left), the cuboctahedral transition state (top right), and both stationary points (bottom). Inversion isomers are lumped together and only minima that correspond to monotonic sequence basin bottoms and also lie in the lowest 2000 are included. One branch is also included for the second-lowest minimum in each case. 
for $\mathrm{LJ}_{55}^{I_{h}}$. A minimum derived from the icosahedron is identified as the second-lowest minimum for $\mathrm{LJ}_{55}^{O_{h}}$ (it does not lie at the end of a monotonic sequence), and this landscape exhibits more low-lying minima connected to the $O_{h}$ minimum by larger, but still relatively small, barriers. The structure of the doubly addressable landscape appears intermediate in character between the graphs obtained for $\mathrm{LJ}_{55}^{I_{h}}$ and $\mathrm{LJ}_{55}^{O_{h}}$.

\section{GLOBAL PROPERTIES OF ADDRESSABLE CLUSTERS}

Two properties were calculated to examine the emergence of characteristics that reflect global features of the underlying potential energy landscape, namely, the heat capacity and a recently introduced measure of frustration. ${ }^{130}$ Here we consider the smaller clusters, where the stationary point databases are probably complete. The heat capacity was calculated using the superposition approach, where the partition function is written as a sum over contributions from the local minima. ${ }^{102,131-135}$ The harmonic approximation was employed for the local vibrational densities of states, corresponding to the standard normal mode analysis. Hence we neglect well anharmonicity, but include the landscape anharmonicity, which derives from the distribution of local minima. ${ }^{113-115}$ Anharmonicity arising from the deviation of local potential wells from the normal mode Hamiltonian generally introduces systematic shifts in heat capacity peaks, since higher energy minima corresponding to a liquid-like phase usually have lower vibrational frequencies. However, it is the effect of landscape entropy that is of interest here, especially in comparing results for changes in the fractional well depth parameter $\alpha$. The partition functions employed in the present work must omit symmetry numbers corresponding to the molecular point group, since the permutational isomers are counted explicitly. The addressable potential lifts some of the usual permutational degeneracies, even when some geometrical symmetry elements are retained in certain stationary points.

Competition between low energy structures with different morphologies separated by high barriers has been associated with "frustration." 109,136 To analyse the degree of frustration in the landscapes of these addressable clusters, we calculated

$$
f(T)=\sum_{\gamma \neq \mathrm{gmin}} p_{\gamma}^{\mathrm{eq}}(T)\left(\frac{V_{\gamma}^{\dagger}-V_{\mathrm{gmin}}}{V_{\gamma}-V_{\mathrm{gmin}}}\right),
$$

where $T$ is the temperature, $p_{\gamma}^{\mathrm{eq}}(T)$ is the equilibrium occupation probability of minimum $\gamma, V_{\text {gmin }}$ signifies the potential
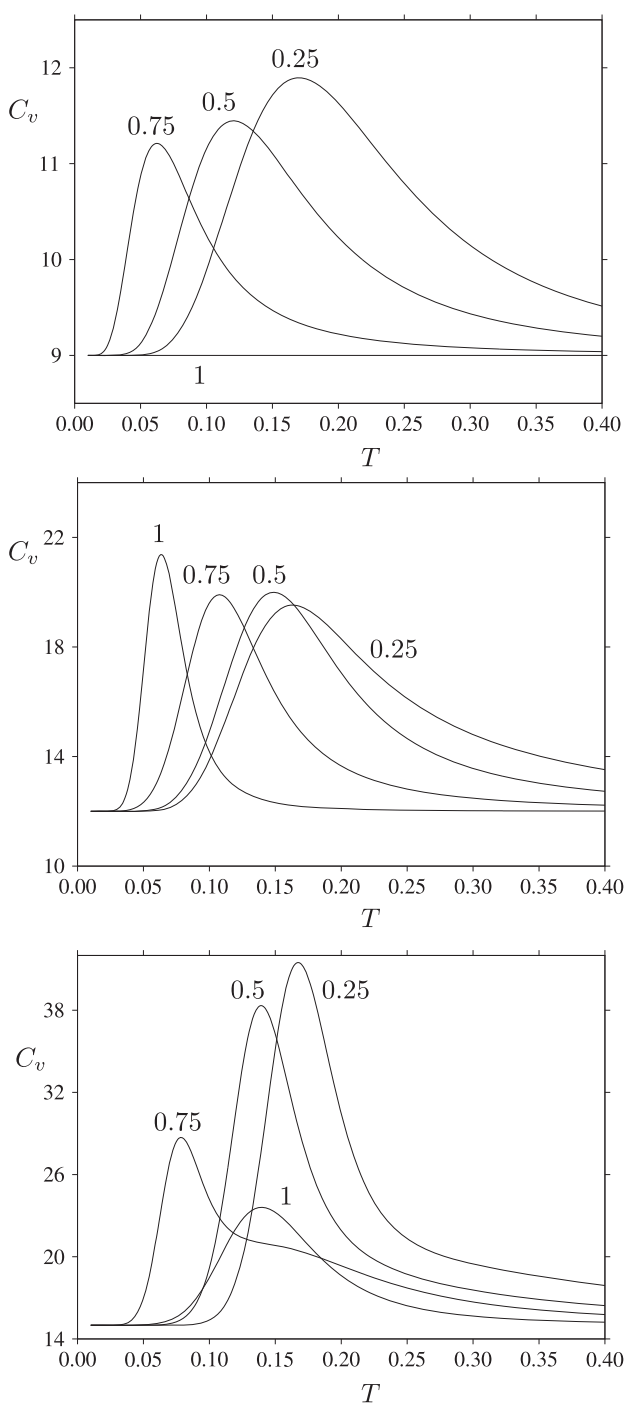
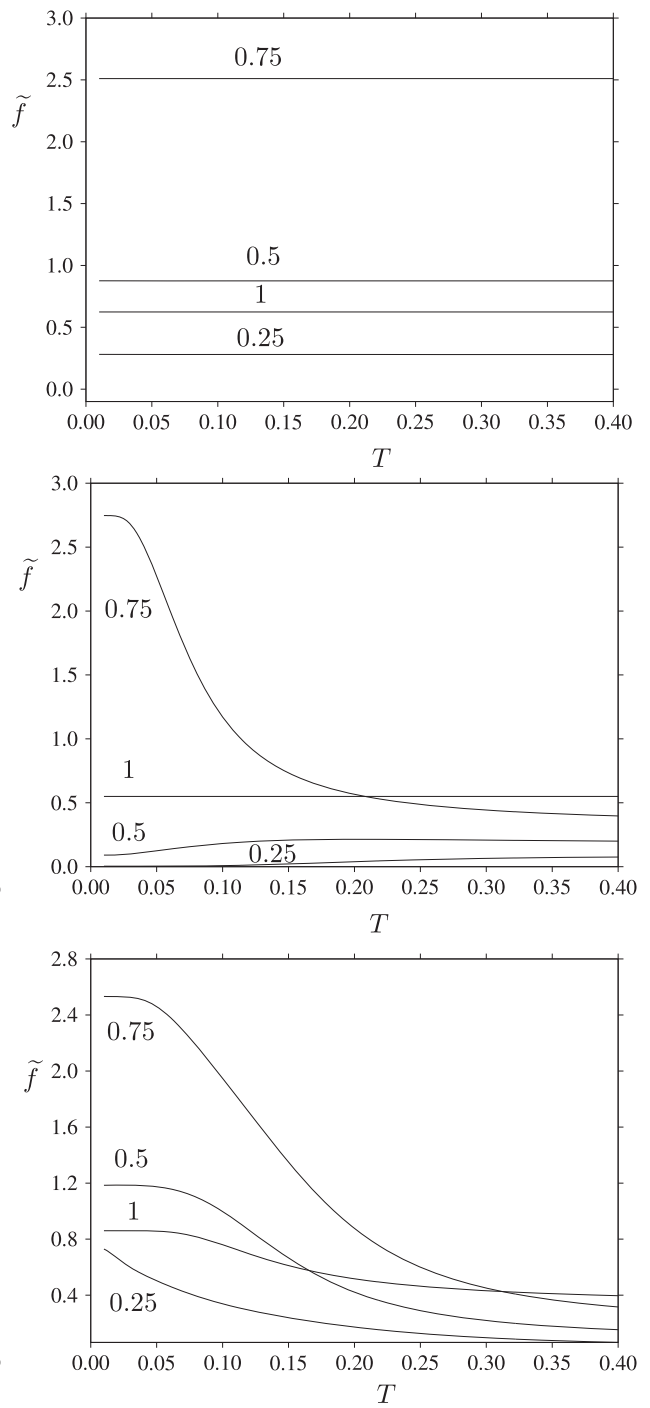

FIG. 7. Heat capacity and frustration index (left and right) as a function of temperature for addressable clusters $\mathrm{LJ}_{5}$, $\mathrm{LJ}_{6}$, and $\mathrm{LJ}_{7}$ (top to bottom). In each case, results are presented for $\alpha$ values of $1,0.75,0.5$, and 0.25 , as marked, corresponding to increasing bias towards the reference structure. $C_{v}$ and $T$ are in reduced units; $\widetilde{f}$ is dimensionless. For $\mathrm{LJ}_{5}$ with $\alpha=1$, all the minima are permutational isomers of the global minimum and the landscape is unfrustrated. 
energy of the lowest minimum in the database, $V_{\gamma}^{\dagger}$ is the potential energy of the highest transition state on the lowest energy path between minimum $\gamma$ and this lowest minimum, and $V_{\gamma}$ is the potential energy of minimum $\gamma . f(T)$ employs barrier thresholds $V_{\gamma}^{\dagger}$ in the same way as a scheme that we introduced in earlier work to help refine databases produced by discrete path sampling to remove artificial frustration. ${ }^{137}$ This approach was, in turn, derived from an analogous index based on free energies that we used earlier, ${ }^{138}$ which extends measures based on stability ${ }^{139}$ and energy gaps ${ }^{140,141}$ by including explicit barrier information.

$p_{\gamma}^{\mathrm{eq}}(T)$ was calculated using harmonic vibrational densities of states, and $V_{\gamma}^{\dagger}$ is the potential energy of the lowest transition state for which a path exists between minimum $\gamma$ and the global minimum. The most insightful index is probably $\widetilde{f}$ calculated from the renormalised probabilities $\widetilde{p}_{\gamma}^{\mathrm{eq}}=p_{\gamma}^{\mathrm{eq}} /\left(1-p_{\mathrm{gmin}}^{\mathrm{eq}}\right)$. This quantity reflects the renormalised relative populations of the minima when the temperature dependence of the global minimum is removed. Here $p_{\text {gmin }}^{\mathrm{eq}}$ is summed over degenerate global minima, i.e., all permutationinversion isomers for $\alpha=1$, and the two enantiomers for $\alpha<1$.

To calculate $\widetilde{f}$, we require databases where the connectivity of the local minima is defined by transition states. We obtained $V_{\gamma}^{\dagger}$ via the superbasin analysis ${ }^{90}$ that yields the disconnectivity graphs, identifying the energy threshold below which the lowest minimum is no longer accessible from minimum $\gamma$. Results for $\widetilde{f}(T)$, which we will refer to as the frustration index, are shown in Figure 7.

The heat capacity peaks shift to higher temperature as $\alpha$ varies through $0.75,0.5$, to 0.25 . This trend reflects the increasing energy gap between the global minimum and the higher energy minima, as expected for the finite system analogue of a first order phase transition. The global minimum is stabilised by potential energy, and the other minima can be viewed collectively as a higher entropy phase-like form. Here the variation in potential energy with $\alpha$ is more significant than changes in the vibrational entropy, consistent with results for clusters containing a dopant atom. ${ }^{122}$ The results for the unperturbed potential can be qualitatively different because of the higher degeneracies. For example, $C_{v}$ for $\mathrm{LJ}_{5}$ is temperature independent, because all the minima are degenerate.

The trends for the frustration index are related to those for $C_{v}$. For $\mathrm{LJ}_{5}^{*}$ the minima split into two sets for $\alpha<1$, and so there is no temperature dependence once the effect of the global minimum is removed. For the other two sizes, $\widetilde{f}(T)$ usually decreases through the $\alpha$ values $0.75,0.5$, to 0.25 , reflecting a decrease in frustration. This observation is consistent with the landscape becoming more biased (funnelled) towards the global minimum. Again, the unperturbed potential behaves differently, because of the unresolved permutational degeneracies, which results in a qualitatively different energy level spectrum.

Hence we see that both $C_{v}$, a key experimental observable, and $\widetilde{f}(T)$, which is readily obtained from the stationary point databases, can provide insight into the global structure of the landscape in a compact form. Experimentally, the yield of a target structure will depend upon the starting conditions, annealing schedule, and the observation time scale. An upper
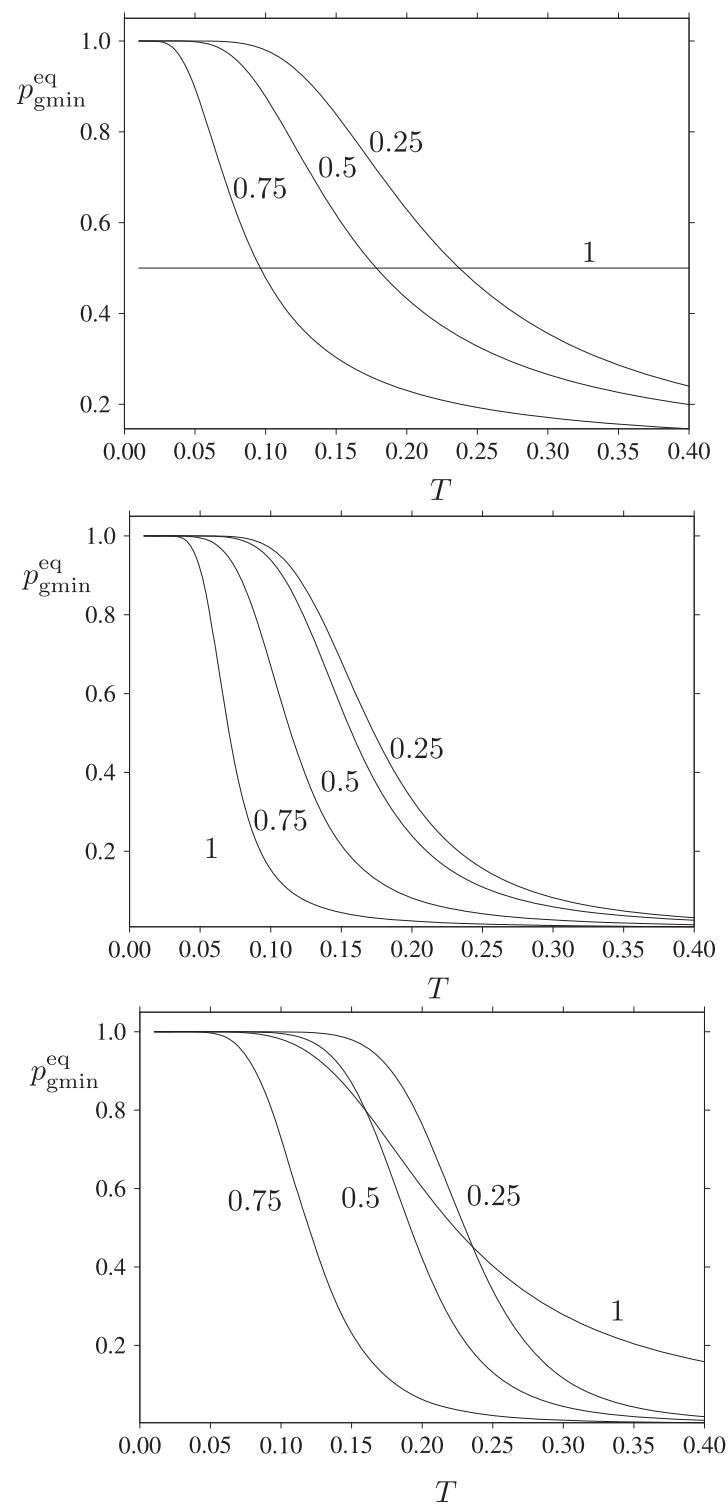

FIG. 8. Equilibrium occupation probability of the global minimum as a function of temperature for addressable clusters $\mathrm{LJ}_{5}, \mathrm{LJ}_{6}$, and $\mathrm{LJ}_{7}$ (top to bottom). In each case, results are presented for $\alpha$ values of $1,0.75,0.5$, and 0.25 , as marked, corresponding to increasing bias towards the reference structure. $T$ is in reduced units.

bound on the yield in the limit of slow enough annealing can be obtained from the equilibrium occupation probability of the global minimum, which is used in the renormalisation of $\widetilde{f}(T)$. This probability is plotted as a function of temperature and $\alpha$ for $\mathrm{LJ}_{5}^{*}, \mathrm{LJ}_{6}^{*}$, and $\mathrm{LJ}_{7}^{*}$ in Figure 8, again using the approximation of harmonic vibrational densities of states. There is a clear trend for $\alpha \neq 1$, with larger equilibrium yields of the target as $\alpha$ decreases.

\section{MULTIPLE TARGET CLUSTERS}

The energy landscapes for selected sizes between two and 120 copies of $\mathrm{LJ}_{5}{ }^{*}$ were first surveyed using basin-hopping global optimisation. Initial runs were conducted using the local rigid body formulation ${ }^{142}$ to sample the configuration space of clusters with particles maintained in their correct addressable sites for the target cluster. A range of values for $\epsilon^{\text {rep }}$ between 


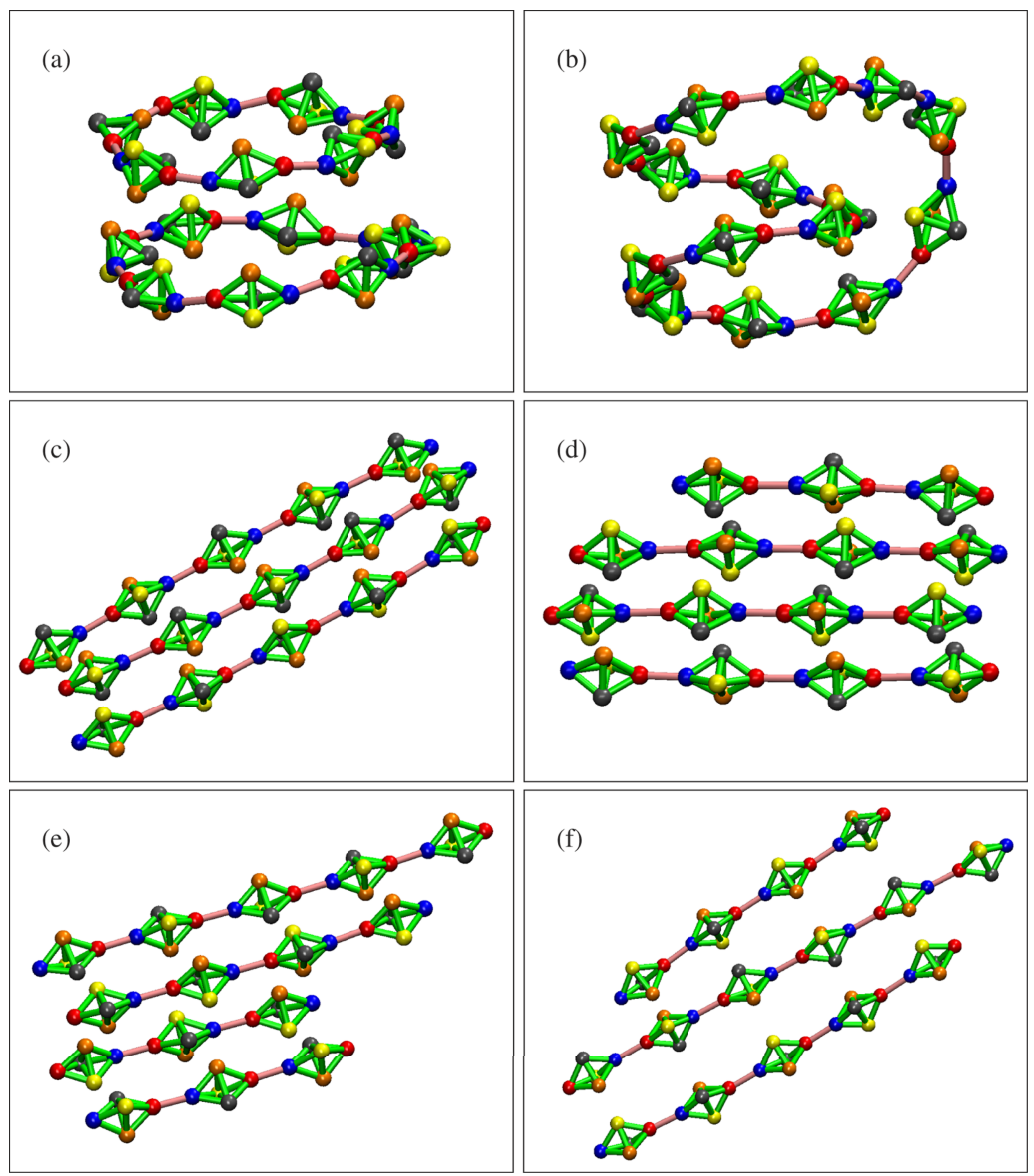

FIG. 9. Some of the structural motifs observed for 15 addressable $\mathrm{LJ}_{5}^{*}$ clusters, identified by green edges within each target, and pink edges between apical atoms to help visualise the overall organisation. Panels (a) and (b) are the lowest and second-lowest minima located in short basin-hopping runs for $\epsilon^{\mathrm{rep}}=6000$. (a) Stacked eight-plus seven-membered rings, (b) a chain of 15 target clusters containing a spiral section. (c) The lowest minimum obtained for $\epsilon^{\mathrm{rep}}=10000$, composed of three parallel chains of five target clusters. Panels (d)-(f) illustrated the lowest minimum and two low-lying minima for $\epsilon^{\text {rep }}=12000$, composed of chains of identifiable target clusters. All these minima correspond to unconstrained relaxation.

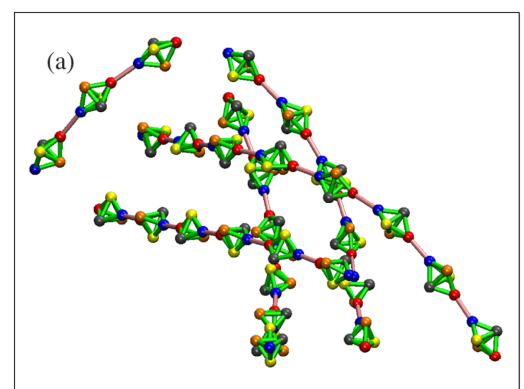

(c)

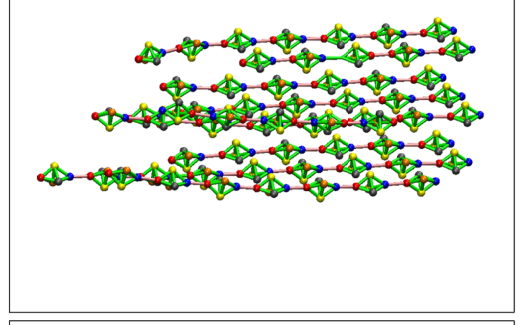

(e)

$$
\text { \& }
$$
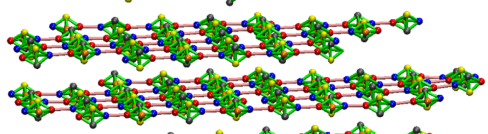

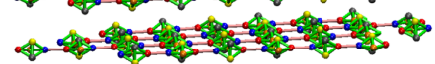

\& $-20-40$

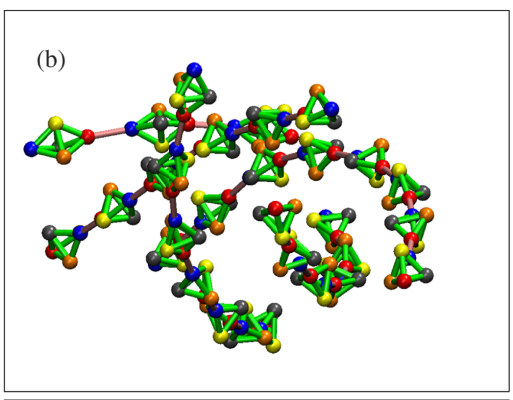

(d)

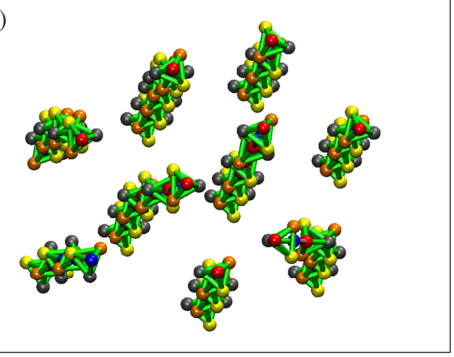

(f)

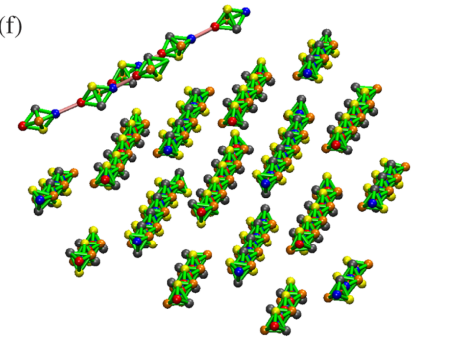

FIG. 10. Two views of the lowest minima obtained in short basin-hopping runs for aggregates of $\mathrm{LJ}_{5}{ }^{*}$ clusters with $\epsilon^{\text {rep }}=15000$ containing [(a) and (b) 30 targets, [(c) and (d)] 60 targets, [(e) and (f)] 120 targets. Green edges are drawn within the distinct target clusters, and pink edges are included between apical atoms to help identify the overall organisation. 
500 and 15000 (in units of $\epsilon$ ) was considered. The lowest five minima obtained in each case with local rigidification were then fully relaxed with all the $\epsilon^{\text {rep }}$ values considered. The lowest minimum for each $\epsilon^{\text {rep }}$ was then used to initiate a new global optimisation run, and this procedure was iterated until the lowest fully relaxed structure in each case was unchanged, which only required a few cycles.

The most systematic tests were conducted for 15 copies of a five-particle addressable cluster with $\alpha=0.1$. For $500<\epsilon^{\text {rep }}<2000$, the lowest minima obtained in unconstrained relaxation for clusters of initially rigid $\mathrm{LJ}_{5}{ }^{*}$ molecules with correctly addressed atoms are quite compact. The identity of the targets is not well preserved in the relaxed aggregates. However, above $\epsilon^{\text {rep }}$ around 3000 the cluster structure is still identifiable after relaxation. The lowest minima for 3000 $<\epsilon^{\text {rep }}<6000$ include stacked rings of size eight plus seven [Figure 9(a)] and nine plus six, and a chain containing a spiral [Figure 9(b)]. For larger values of $\epsilon^{\text {rep }}$, the dominant motifs are parallel or partly bent chains, as shown in Figures 9(c)-9(f).

These motifs are also found when more copies of the target are included; some examples are shown in Figure 10. The lowest minima located in short basin-hopping runs for 30,60 , and 120 copies of the target involve parallel or bent chains. All of these structures were fully relaxed with no constraints. Locating the true global minima will require much longer runs, but the structures identified so far strongly suggest that all the favourable aggregates for parameters in this range will contain arrays of chains and perhaps rings. The possibility of exploiting hierarchical design to produce complex morphologies clearly warrants further investigation. To provide some indication of the likely complexity encoded by such aggregates, we conclude this preliminary survey with a detailed analysis of the landscape for the $\mathrm{LJ}_{5}{ }^{*}$ dimer.

Two copies of $\mathrm{LJ}_{5}{ }^{*}$ were first considered, with each one treated as a local rigid body based on the target global minimum. The number of distinct structures for local minima on this simplified landscape falls from three for $\epsilon^{\mathrm{rep}}=500$ to two for $\epsilon^{\mathrm{rep}}=3000$, and then a single minimum for $\epsilon^{\text {rep }}=12000$ and above, where a competing sideby-side configuration disappears. The end-to-end minimum with apical atoms in a line emerges at $\epsilon^{\mathrm{rep}}=6000$. Here we have not counted minima with positive energies, where the two rigidified $\mathrm{LJ}_{5}{ }^{*}$ clusters interpenetrate. A detailed analysis of the unconstrained landscape was then performed for $\epsilon^{\text {rep }}=12000$ using the discrete path sampling tools encoded in our OPTIM ${ }^{143}$ and PATHSAMPLE ${ }^{94}$ programs.

There are three structures corresponding to dimers of correctly addressed targets on the unconstrained landscape. Two are end-to-end configurations, the lowest with staggered apical atoms (energy $-18.10308 \epsilon$ ), the other with the three equatorial atoms of the two trigonal bipyramids eclipsed (energy $-18.06800 \epsilon$ ). A side-by-side structure lies slightly higher in energy $(-18.03798 \epsilon)$. End-toend configurations with wrongly addressed atoms in the two trigonal bipyramids appear about $0.7 \epsilon$ higher in energy. Dimers where the components are not describable as trigonal bipyramids appear about $1.8 \epsilon$ above the global minimum.
The disconnectivity graph where all permutational isomers are distinguished, with enantiomers lumped together, is shown in Figure 11. The 16 subfunnels correspond to the exchange of particles with the same address. There are $2^{5}=32$ permutations for every minimum, and the 16 funnels each contain two permutational isomers of the global minimum along with the corresponding enantiomers. These two permutational isomers are related by the exchange of all pairs of particles with the same address; the barrier to this one-step rearrangement is $0.05846 \epsilon$.

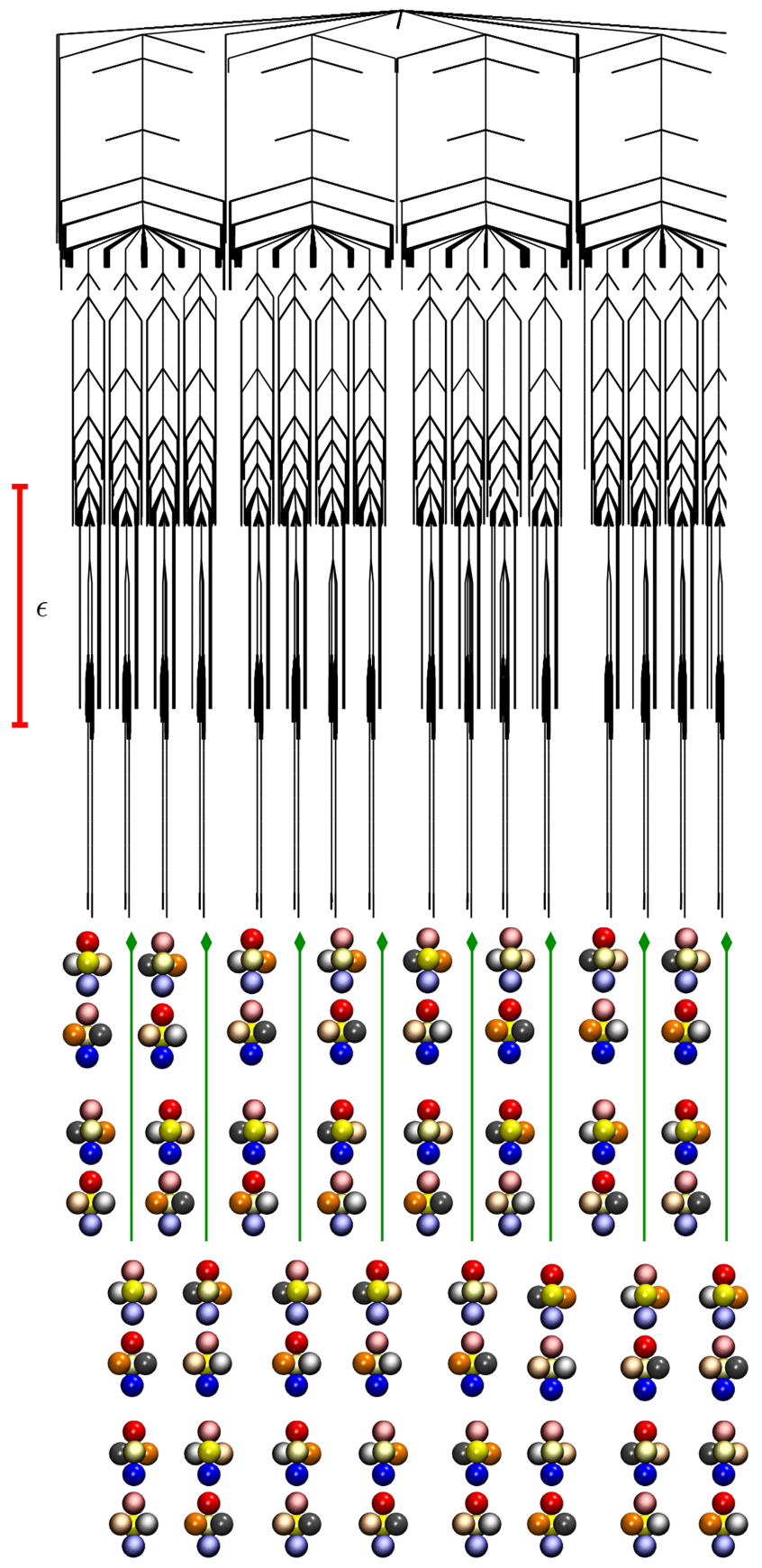

FIG. 11. Disconnectivity graph for the $\mathrm{LJ}_{5}{ }^{*}$ dimer with $\epsilon^{\mathrm{rep}}=12000$. The 32 distinct permutational isomers of the global minimum appear in pairs at the bottom of the 16 subfunnels. The equivalent pairs of atoms are coloured bright and pale shades of blue, red, orange, silver, and yellow, using a consistent numbering scheme. 


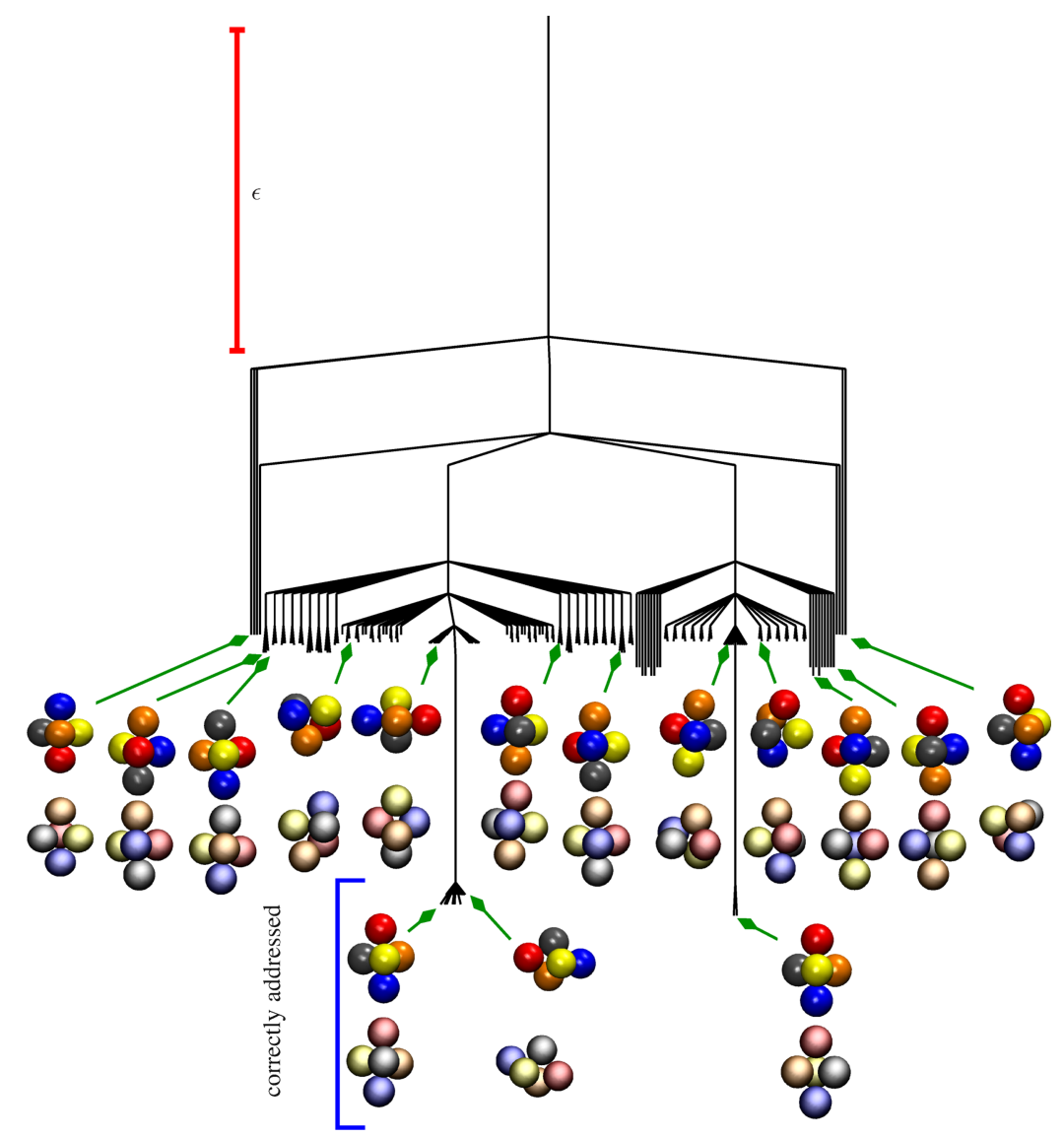

FIG. 12. Disconnectivity graph for one subfunnel for the $\mathrm{LJ}_{5}{ }^{*}$ dimer with $\epsilon^{\mathrm{rep}}=12000$, including permutational isomers of the 17 lowest energy structures. The three dimers composed of distinguishable correctly addressed target clusters are well separated from minima with address errors. Twelve of the fourteen structures with one or both components containing address errors are also illustrated. The equivalent pairs of atoms are coloured bright and pale shades of blue, red, orange, silver, and yellow, using a consistent numbering scheme.
The hierarchical organisation of the landscape is clear, with the 16 funnels corresponding to subsets of local minima that are connected in groups of four via a barrier of around $2.86596 \epsilon$. Both permutational isomers of the global minimum are illustrated for each funnel in Figure 11, with the pairs of equivalent atoms coloured bright and pale shades of blue, red, orange, yellow, and silver in a consistent numbering scheme. The database that underlies this graph contains 37643 minima (37121 in the largest connected component) and 184217 transition states. To check the convergence of the sampling, symmetry was not exploited, and the resulting hierarchical structure emerged systematically as new connections between minima was located.

A magnification of the low-energy region of one subfunnel is shown in Figure 12. The graph is based on the lowest 204 minima in this region with 17 distinct structures, which includes all those with distinguishable correctly addressed target clusters, along with some higher energy configurations corresponding to wrongly addressed components; there is a clear energetic separation between these sets.

The transition state that permits interchange between all permutational isomers of the global minimum corresponds to an overall barrier of $3.75623 \epsilon$. The yield of correctly addressed targets will depend on the energy gap between these targets and the lowest alternative minima. The relatively large gap observed here means that dimers composed of the correct targets are thermodynamically favourable and kinetically accessible over a significant range of temperature (or microcanonical total energy).

\section{CONCLUSIONS}

The present results show that altering the strength of interparticle interactions to favour contacts that are present in a reference structure can provide a powerful design tool for addressable structures. Similar constructions have been used before to simplify the analysis of biomolecules, and a generalisation to clusters and soft and condensed matter is straightforward. The present approach could be used for pairwise and many-body potentials, and the biasing could be tuned more extensively using a range of well depth and cutoff parameters.

In fact, a simple implementation with a single cutoff and a uniform reduction in the strength of non-nearest-neighbour interactions is sufficient to produce addressable clusters for the systems considered here. Landscapes with increasing selforganising characteristics are obtained as the bias changes systematically. It is also possible to define target structures corresponding to a relatively high energy transition state with a completely different morphology in the 55-atom cluster and to design a doubly addressable system for this geometry and a permutational isomer of the global minimum.

For multiple copies of the target cluster, introducing a repulsive term between particles with the same address can produce aggregates with distinct copies of the target. As the repulsive parameter increases, the favourable morphologies for these aggregates include rings and chains. Realising these 
structures experimentally will require a mapping from the model potential, in terms of competing energy and length scales, to details of the intermolecular potential for the building blocks involved. We have previously exploited such principles to suggest designs for helical assemblies. ${ }^{129,144,145}$

Visualising the underlying potential energy landscape using disconnectivity graphs provides insight into the evolution with increasing bias. Global thermodynamic properties are reflected in the heat capacity, while the frustration index includes information about barrier heights, and hence kinetic accessibility. Identifying structures that are only directly connected to higher-lying minima as biminima, provides a convenient way to simplify the disconnectivity graphs for larger databases. These tools could play an important role in future design efforts for addressable systems, including multifunctional properties that we associate with multifunnel landscapes. $^{146}$

\section{ACKNOWLEDGMENTS}

I am very grateful to Brooke Husic, Dmitri Schebarchov, and Aleks Reinhardt for comments on the first draft of this manuscript, to John Morgan for his assistance with some of the graphics, and to Professor Robert Jack for insight into previous work on glassy systems.

${ }^{1}$ Y. Ke, L. L. Ong, W. M. Shih, and P. Yin, Science 338, 1177 (2012).

${ }^{2}$ A. Reinhardt and D. Frenkel, Phys. Rev. Lett. 112, 238103 (2014).

${ }^{3}$ W. M. Jacobs, A. Reinhardt, and D. Frenkel, Proc. Natl. Acad. Sci. U. S. A. 112, 6313 (2015).

${ }^{4}$ G. Meng, N. Arkus, M. P. Brenner, and V. N. Manoharan, Science 327, 560 (2010).

${ }^{5}$ J. C. Crocker, Science 327, 535 (2010).

${ }^{6}$ D. J. Wales, ChemPhysChem 11, 2491 (2010).

${ }^{7}$ S. Hormoz and M. P. Brenner, Proc. Natl. Acad. Sci. U. S. A. 108, 5193 (2011).

${ }^{8}$ D. Frenkel and D. J. Wales, Nat. Mater. 10, 410 (2011).

${ }^{9}$ F. Calvo, J. P. K. Doye, and D. J. Wales, Nanoscale 4, 1085 (2012).

${ }^{10} \mathrm{~J}$. W. Collins, "Self-assembly of colloidal spheres with specific interactions," Ph.D. dissertation (Harvard University, 2014), http://nrs.harvard.edu/urn-3:hul.instrepos:12274201.

${ }^{11}$ B. Derrida, Phys. Rev. B 24, 2613 (1981).

${ }^{12}$ J. D. Bryngelson and P. G. Wolynes, Proc. Natl. Acad. Sci. U. S. A. 84, 7524 (1987)

${ }^{13}$ J. E. Jones and A. E. Ingham, Proc. R. Soc. A 107, 636 (1925).

${ }^{14}$ Y. Ueda, H. Taketomi, and N. Gō, Biopolymers 17, 1531 (1978).

${ }^{15}$ S. Franz and G. Parisi, Phys. Rev. Lett. 79, 2486 ( 1997).

${ }^{16}$ L. Berthier and R. L. Jack, Phys. Rev. Lett. 114, 205701 (2015).

${ }^{17}$ R. L. Jack and J. P. Garrahan, Phys. Rev. Lett. 116, 055702 (2016).

${ }^{18}$ C. J. Fullerton and R. L. Jack, Phys. Rev. Lett. 112, 255701 (2014).

${ }^{19}$ R. L. Jack and C. J. Fullerton, Phys. Rev. E 88, 042304 (2013).

${ }^{20}$ L. Berthier and W. Kob, Phys. Rev. E 85, 011102 (2012).

${ }^{21}$ W. Kob and L. Berthier, Phys. Rev. Lett. 110, 245702 (2013).

${ }^{22}$ S. Karmakar and G. Parisi, Proc. Natl. Acad. Sci. U. S. A. 110, 2752 (2013).

${ }^{23}$ C. Cammarota and G. Biroli, Euro. Phys. Lett. 98, 16011 (2012).

${ }^{24}$ C. Cammarota and G. Biroli, Proc. Natl. Acad. Sci. U. S. A. 109, 8850 (2012).

${ }^{25}$ C. Cammarota and G. Biroli, J. Chem. Phys. 138, 12A547 (2013).

${ }^{26}$ K. Kim, Europhys. Lett. 61, 790 (2003).

${ }^{27}$ M. Ozawa, W. Kob, A. Ikeda, and K. Miyazaki, Proc. Natl. Acad. Sci. U. S. A. 112, 6914 (2015).

${ }^{28}$ C. Cammarota, G. Gradenigo, and G. Biroli, Phys. Rev. Lett. 111, 107801 (2013).

${ }^{29}$ Y. W. Li, W. S. Xu, and Z. Y. Sun, J. Chem. Phys. 140, 124502 (2014).

${ }^{30}$ A. Cavagna, T. S. Grigera, and P. Verrocchio, J. Chem. Phys. 136, 204502 (2012).

${ }^{31}$ A. Cavagna, T. S. Grigera, and P. Verrocchio, Phys. Rev. Lett. 98, 187801 (2007).
${ }^{32}$ C. Cammarota and A. Cavagna, J. Chem. Phys. 127, 214703 (2007).

${ }^{33}$ G. Biroli, J.-P. Bouchaud, A. Cavagna, T. S. Grigera, and P. Verrocchio, Nat. Phys. 4, 771 (2008).

${ }^{34}$ C. Cammarota, A. Cavagna, G. Gradenigo, T. S. Grigera, and P. Verrocchio, J. Stat. Mech.: Theory Exp. 2009, L12002.

${ }^{35}$ A. Cavagna, T. S. Grigera, and P. Verrocchio, J. Chem. Phys. 136, 204502 (2012).

${ }^{36}$ L. Berthier, P. Charbonneau, and S. Yaida, J. Chem. Phys. 144, 024501 (2016).

${ }^{37}$ S. Yaida, L. Berthier, P. Charbonneau, and G. Tarjus, Phys. Rev. E 94, 032605 (2016).

${ }^{38}$ M. M. Tirion, Phys. Rev. Lett. 77, 1905 (1996).

${ }^{39}$ F. Tama and Y. H. Sanejouand, Protein Eng., Des. Sel. 14, 1 (2001).

${ }^{40}$ A. R. Atilgan, S. R. Durell, R. L. Jernigan, M. C. Demeril, O. Keskin, and I. Bahar, Biophys. J. 80, 505 (2001).

${ }^{41}$ M. K. Kim, G. S. Chirikjan, and R. L. Jernigan, J. Mol. Graphics Modell. 21, 151 (2002).

${ }^{42}$ D. Ming, Y. Kong, M. A. Lambert, Z. Huang, and J. Ma, Proc. Natl. Acad. Sci. U. S. A. 99, 8620 (2002).

${ }^{43}$ M. Delarue and Y. H. Sanejouand, J. Mol. Biol. 320, 1011 (2002).

${ }^{44}$ M. Ikeguchi, J. Ueno, M. Sato, and A. Kidera, Phys. Rev. Lett. 94, 078102 (2002).

${ }^{45}$ W. Zheng and S. Doniach, Proc. Natl. Acad. Sci. U. S. A. 100, 13253 (2003).

${ }^{46}$ N. Reuter, K. Hinsen, and J. J. Lacapère, Biophys. J. 85, 2186 (2003).

${ }^{47}$ C. Xu, D. Tobi, and I. Bahar, J. Mol. Biol. 333, 153 (2003).

${ }^{48}$ F. Tama, M. Valle, J. Franks, and C. L. Brooks, Proc. Natl. Acad. Sci. U. S. A. 100, 9319 (2003).

${ }^{49}$ P. Maragakis and M. Karplus, J. Mol. Biol. 352, 807 (2005).

${ }^{50}$ I. Bahar and A. J. Rader, Curr. Opin. Struct. Biol. 15, 586 (2005).

${ }^{51}$ P. C. Whitford, O. Miyashita, Y. Levy, and J. N. Onuchic, J. Mol. Biol. 366, 1661 (2007).

${ }^{52}$ W. Zheng, B. R. Brooks, and G. Hummer, Proteins: Struct., Funct., Bioinf. 69, 43 (2007).

${ }^{53}$ A. Korkut and W. A. Hendrickson, Proc. Natl. Acad. Sci. U. S. A. 106, 15667 (2009).

${ }^{54}$ M. Lu and J. Ma, in Energy Flow in Proteins, edited by D. Leitner and J. Straub (CRC Press, Boca Raton, 2009), pp. 229-245.

${ }^{55}$ C. Peng, L. Zhang, and T. Head-Gordon, Biophys. J. 98, 2356 (2010).

${ }^{56}$ P. Batista, C. Robert, J. D. Marechal, M. Hamida-Rebai, P. Pascutti, P. Bisch, and D. Perahia, Phys. Chem. Chem. Phys. 12, 2850 (2010).

${ }^{57}$ R. B. Best, Y.-G. Chen, and G. Hummer, Structure 13, 1755 (2005).

${ }^{58}$ K.-i. Okazaki, N. Koga, S. Takada, J. N. Onuchic, and P. G. Wolynes, Proc. Natl. Acad. Sci. U. S. A. 103, 11844 (2006).

${ }^{59}$ K.-i. Okazaki and S. Takada, Proc. Natl. Acad. Sci. U. S. A. 105, 11182 (2008).

${ }^{60} \mathrm{Q}$. Lu and J. Wang, J. Am. Chem. Soc. 130, 4772 (2008).

${ }^{61}$ Z.-Z. Lai, Q. Lu, and J. Wang, J. Phys. Chem. B 115, 4147 (2011).

${ }^{62}$ A. Murugan, Z. Zeravcic, M. P. Brenner, and S. Leibler, Proc. Natl. Acad. Sci. U. S. A. 112, 54 (2015).

${ }^{63}$ D. Morphew and D. Chakrabarti, Nanoscale 7, 8343 (2015).

${ }^{64}$ M.-P. Valignat, O. Theodoly, J. C. Crocker, W. B. Russel, and P. M. Chaikin, Proc. Natl. Acad. Sci. U. S. A. 102, 4225 (2005).

${ }^{65}$ P. L. Biancaniello, A. J. Kim, and J. C. Crocker, Phys. Rev. Lett. 94, 058302 (2005).

${ }^{66}$ A. J. Kim, P. L. Biancaniello, and J. C. Crocker, Langmuir 22, 1991 (2006).

${ }^{67}$ C. A. Mirkin, R. L. Letsinger, R. C. Mucic, and J. J. Storhoff, Nature 382, 607 (1996).

${ }^{68}$ A. P. Alivisatos, K. P. Johnsson, X. Peng, T. E. Wilson, C. J. Loweth, M. P. Bruchez, and P. G. Schultz, Nature 382, 609 (1996).

${ }^{69}$ S. Y. Park, A. K. R. Lytton-Jean, B. Lee, S. Weigand, G. C. Schatz, and C. A. Mirkin, Nature 451, 553 (2008).

${ }^{70}$ D. Nykypanchuk, M. M. Maye, D. van der Lelie, and O. Gang, Nature 451, 549 (2008).

${ }^{71}$ P. W. K. Rothemund, Nature 440, 297 (2006).

${ }^{72}$ S. M. Douglas, H. Dietz, T. Liedl, B. Högberg, F. Graf, and W. M. Shih, Nature 459, 414 (2009).

${ }^{73}$ B. Wei, M. Dai, and P. Yin, Nature 485, 623 (2012).

${ }^{74}$ T. Wang, R. Sha, R. Dreyfus, M. E. Leunissen, C. Maass, D. J. Pine, P. M. Chaikin, and N. C. Seeman, Nature 478, 225 (2011).

${ }^{75}$ Y.-T. Lai, N. P. King, and T. O. Yeates, Trends in Cell Biol. 22, 653 (2012).

${ }^{76}$ N. P. King, J. B. Bale, W. Sheffler, D. E. McNamara, S. Gonen, T. Gonen, T. O. Yeates, and D. Baker, Nature 510, 103 (2014). 
${ }^{77}$ F. Noé and S. Fischer, Curr. Opin. Struct. Biol. 18, 154 (2008).

${ }^{78}$ D. Prada-Gracia, J. Gómez-Gardenes, P. Echenique, and F. Fernando, PLoS Comput. Biol. 5, e1000415 (2009).

${ }^{79}$ D. J. Wales, Curr. Opin. Struct. Biol. 20, 3 (2010).

${ }^{80}$ Z. Li and H. A. Scheraga, Proc. Natl. Acad. Sci. U. S. A. 84, 6611 (1987).

${ }^{81}$ Z. Li and H. A. Scheraga, J. Mol. Struct. 179, 333 (1988).

${ }^{82}$ D. J. Wales and J. P. K. Doye, J. Phys. Chem. A 101, 5111 (1997).

${ }^{83}$ S. A. Trygubenko and D. J. Wales, J. Chem. Phys. 120, 2082 (2004).

${ }^{84}$ G. Henkelman and H. Jónsson, J. Chem. Phys. 113, 9978 (2000).

${ }^{85}$ G. Henkelman, B. P. Uberuaga, and H. Jónsson, J. Chem. Phys. 113, 9901 (2000).

${ }^{86}$ L. J. Munro and D. J. Wales, Phys. Rev. B 59, 3969 (1999).

${ }^{87}$ G. Henkelman and H. Jónsson, J. Chem. Phys. 111, 7010 (1999).

${ }^{88}$ Y. Kumeda, L. J. Munro, and D. J. Wales, Chem. Phys. Lett. 341, 185 (2001).

${ }^{89}$ J. N. Murrell and K. J. Laidler, Trans. Faraday Soc. 64, 371 (1968).

${ }^{90}$ O. M. Becker and M. Karplus, J. Chem. Phys. 106, 1495 (1997).

${ }^{91}$ D. J. Wales, M. A. Miller, and T. R. Walsh, Nature 394, 758 (1998).

${ }^{92}$ D. J. Wales, Mol. Phys. 100, 3285 (2002).

${ }^{93}$ D. J. Wales, Mol. Phys. 102, 891 (2004).

${ }^{94}$ D. J. Wales, Pathsample: A program for generating connected stationary point databases and extracting global kinetics, http://www-wales. ch.cam.ac.uk/software.html.

${ }^{95}$ C. G. Broyden, IMA J. Appl. Math. 6, 76 (1970).

${ }^{96}$ R. Fletcher, Comput. J. 13, 317 (1970).

${ }^{97}$ D. Goldfarb, Math. Comput. 24, 23 (1970).

${ }^{98}$ D. F. Shanno, Math. Comput. 24, 647 (1970).

${ }^{99}$ A. L. Mackay, Acta Crystallogr. 15, 916 (1962).

${ }^{100}$ W. N. Lipscomb, Science 153, 373 (1966).

${ }^{101}$ D. J. Wales, L. J. Munro, and J. P. K. Doye, J. Chem. Soc., Dalton Trans. 1996, 611-623.

${ }^{102}$ D. J. Wales, Energy Landscapes (Cambridge University Press, Cambridge, 2003).

${ }^{103}$ F. G. Amar and R. S. Berry, J. Chem. Phys. 85, 5943 (1986).

${ }^{104}$ M. K. Gilson and K. K. Irikura, J. Phys. Chem. B 114, 16304 (2010).

${ }^{105}$ D. J. Wales and P. Salamon, Proc. Natl. Acad. Sci. U. S. A. 111, 617 (2014).

${ }^{106}$ M. E. Cates and V. N. Manoharan, Soft Matter 11, 6538 (2015).

${ }^{107}$ D. J. Wales and T. V. Bogdan, J. Phys. Chem. B 110, 20765 (2006).

${ }^{108}$ M. Karplus and A. Šali, Curr. Opin. Struct. Biol. 5, 58 (1995).

${ }^{109}$ J. D. Bryngelson, J. N. Onuchic, N. D. Socci, and P. G. Wolynes, Proteins: Struct., Funct., Genet. 21, 167 (1995).

${ }^{110}$ J. N. Onuchic, P. G. Wolynes, Z. Luthey-Schulten, and N. D. Socci, Proc. Natl. Acad. Sci. U. S. A. 92, 3626 (1995).

${ }^{111}$ J. N. Onuchic, H. Nymeyer, A. E. García, J. Chahine, and N. D. Socci, Adv. Protein Chem. 53, 87 (2000).

${ }^{112}$ P. E. Leopold, M. Montal, and J. N. Onuchic, Proc. Natl. Acad. Sci. U. S. A. 89, 8721 (1992).

${ }^{113}$ F. Sciortino, W. Kob, and P. Tartaglia, J. Phys.: Condens. Matter 12, 6525 (2000).

${ }^{114}$ T. V. Bogdan, D. J. Wales, and F. Calvo, J. Chem. Phys. 124, 044102 (2006).

${ }^{115}$ D. J. Wales, Chem. Phys. Lett. 584, 1 (2013).
${ }^{116}$ R. S. Berry and R. Breitengraser-Kunz, Phys. Rev. Lett. 74, 3951 (1995).

${ }^{117}$ R. E. Kunz and R. S. Berry, J. Chem. Phys. 103, 1904 (1995).

${ }^{118}$ J. P. Rose and R. S. Berry, J. Chem. Phys. 98, 3246 (1993).

${ }^{119}$ K. D. Ball, R. S. Berry, R. E. Kunz, F.-Y. Li, A. Proykova, and D. J. Wales, Science 271, 963 (1996).

${ }^{120}$ D. Schebarchov and D. J. Wales, Phys. Rev. Lett. 113, 156102 (2014).

${ }^{121}$ D. Schebarchov and D. J. Wales, Phys. Chem. Chem. Phys. 17, 28331 (2015).

${ }^{122}$ B. E. Husic, D. Schebarchov, and D. J. Wales, Nanoscale 8, 18326 (2016).

${ }^{123}$ J. P. K. Doye, M. A. Miller, and D. J. Wales, J. Chem. Phys. 110, 6896 (1999).

${ }^{124}$ J. P. Neirotti, F. Calvo, D. L. Freeman, and J. D. Doll, J. Chem. Phys. 112, 10340 (2000).

${ }^{125}$ M. Picciani, M. Athenes, J. Kurchan, and J. Tailleur, J. Chem. Phys. 135, 034108 (2011).

${ }^{126}$ V. A. Sharapov and V. A. Mandelshtam, J. Phys. Chem. A 111, 10284 (2007).

${ }^{127}$ F. Calvo, Phys. Rev. E 82, 046703 (2010).

${ }^{128}$ D. J. Wales, Phil. Trans. Roy. Soc. A, 370, 2877 (2012).

${ }^{129}$ D. Chakrabarti and D. J. Wales, Soft Matter 7, 2325 (2011).

${ }^{130}$ V. K. de Souza, J. Stevenson, J. D. Farrell, C. P. Goodrich, S. P. Niblett, and D. J. Wales, "Defining and quantifying frustration in the energy landscape: Applications to atomic and molecular clusters, biomolecules, jammed and glassy systems," J. Chem. Phys. (submitted).

${ }^{131}$ F. H. Stillinger and T. A. Weber, Science 225, 983 (1984).

${ }^{132}$ D. J. Wales, Mol. Phys. 78, 151 (1993).

${ }^{133}$ F. H. Stillinger, Science 267, 1935 (1995).

${ }^{134}$ B. Strodel and D. J. Wales, Chem. Phys. Lett. 466, 105 (2008).

${ }^{135}$ V. A. Sharapov, D. Meluzzi, and V. A. Mandelshtam, Phys. Rev. Lett. 98, 105701 (2007).

${ }^{136}$ J. N. Onuchic, Z. Luthey-Schulten, and P. G. Wolynes, Annu. Rev. Phys. Chem. 48, 545 (1997).

${ }^{137}$ B. Strodel, C. S. Whittleston, and D. J. Wales, J. Am. Chem. Soc. 129, 16005 (2007).

${ }^{138}$ J. M. Carr and D. J. Wales, J. Phys. Chem. B 112, 8760 (2008).

${ }^{139}$ A. Godzik, A. Kolinski, and J. Skolnick, J. Comput.-Aided Mol. Des. 7, 397 (1993).

${ }^{140}$ D. U. Ferreiro, J. A. Hegler, E. A. Komives, and P. G. Wolynes, Proc. Natl. Acad. Sci. U. S. A. 104, 19819 (2007).

${ }^{141}$ D. U. Ferreiro, J. A. Hegler, E. A. Komives, and P. G. Wolynes, Proc. Natl. Acad. Sci. U. S. A. 108, 3499 (2011).

${ }^{142}$ H. Kusumaatmaja, C. S. Whittleston, and D. J. Wales, J. Chem. Theory Comput. 8, 5159 (2012).

${ }^{143}$ D. J. Wales, Optim: A program for geometry optimisation and pathway calculations, http://www-wales.ch.cam.ac.uk/software.html.

${ }^{144}$ D. Chakrabarti, S. N. Fejer, and D. J. Wales, Proc. Natl. Acad. Sci. U. S. A. 106, 20164 (2009).

${ }^{145}$ S. N. Fejer, D. Chakrabarti, H. Kusumaatmaja, and D. J. Wales, Nanoscale 6, 9448 (2014).

${ }^{146}$ Y. Chebaro, A. J. Ballard, D. Chakraborty, and D. J. Wales, Sci. Rep. 5, 10386 (2015). 